\title{
Optimal axes of Siberian snakes for polarized proton acceleration
}

\author{
Georg H. Hoffstaetter \\ Department of Physics, Cornell University, Ithaca, New York, USA
}

(Received 30 April 2004; published 17 December 2004)

\begin{abstract}
Accelerating polarized proton beams and storing them for many turns can lead to a loss of polarization when accelerating through energies where a spin rotation frequency is in resonance with orbit oscillation frequencies. First-order resonance effects can be avoided by installing Siberian snakes in the ring, devices which rotate the spin by $180^{\circ}$ around the snake axis while not changing the beam's orbit significantly. For large rings, several Siberian snakes are required. Here a criterion will be derived that allows one to find an optimal choice for the orientation of the snake axes. Rings with superperiod four are analyzed in detail, and the HERA proton ring is used as an example for approximate fourfold symmetry. The proposed arrangement of Siberian snakes matches their effects so that all spin-orbit coupling integrals vanish at all energies and therefore there is no first-order spinorbit coupling at all for this choice, which I call snake matching. It will be shown that in general at least eight Siberian snakes are needed and that there are exactly four possibilities to arrange their axes. When the betatron phase advance between snakes is chosen suitably, four Siberian snakes can be sufficient. Since the spin motion depends on a particle's trajectory, protons at different phase space positions generally have different spin directions. The time averaged polarization at each phase space point is parallel to the invariant spin field, and the spread of this spin field limits the polarization that can be stored. By the here presented choice of Siberian snakes this limit is completely eliminated up to first order in the transverse phase space coordinates. The invariant spin field and the amplitude-dependent spin tune are also computed without linearization to show the advantages of this choice of snakes. Ultimately, the goal of snake matching is to reduce the loss of polarization during the acceleration of the beam. To show that a favorable choice of snakes has been found, polarized protons are tracked for part of HERA- $p$ 's acceleration cycle which shows that polarization is preserved best for the here proposed arrangement of Siberian snakes.
\end{abstract}

DOI: 10.1103/PhysRevSTAB.7.121001

PACS numbers: 29.27.Hj, 29.20.-c, 41.75.Lx

\section{INTRODUCTION}

The design orbit spin direction $\vec{n}_{0}$ at some azimuth $\theta=$ $2 \pi l / L$ along a storage ring of length $L$ describes the spin direction for the design orbit which is periodic from turn to turn, where $l$ is the path length along the design orbit. The design orbit spin tune $\nu_{0}$ describes the number of times a spin has rotated around $\vec{n}_{0}(\theta)$ during one turn around the ring.

Spin-orbit resonances in high-energy accelerators arise when the electromagnetic fields on synchrobetatron trajectories cause disturbances of the spin's motion which build up coherently from turn to turn.

In a flat ring, for instance, an initially vertical spin of a particle traveling on the design orbit remains vertical during particle motion. On a vertical betatron trajectory the particle traverses horizontal fields in quadrupoles and the spin no longer remains vertical. This disturbance of spin motion due to the betatron motion is described by the spin-orbit coupling integrals [1]

$$
I^{ \pm}=\int_{0}^{2 \pi} k_{y} e^{i\left[-\Psi(\theta) \pm \Phi_{y}(\theta)\right]} d \theta,
$$

where $k_{y}=k \sqrt{\beta_{y}}$ with the quadrupole strength $k$ and the vertical beta function $\beta_{y}$, and $\Psi$ is the phase advance of the spin rotation around the vertical and $\Phi_{y}$ is the vertical betatron phase. For ease of notation a constant factor which is sometimes multiplied to this integral is not indicated here.

The $I^{ \pm}$tend to be especially big when the spin disturbance in every FODO cell of a ring adds up coherently [2].

In an approximation that is of first order in transverse phase space coordinates, these integrals yield the following important information: If all spin-orbit coupling integrals vanish, all initially vertical spins are again vertical after one turn, although they have traveled along different betatron trajectories. The ring is then called spin matched or spin transparent.

In this paper it will be analyzed how Siberian snakes can be used to make a storage ring spin transparent at all energies.

When a beam is polarized, it has a polarization direction $\vec{f}(\vec{z}, j)$ at each phase space position $\vec{z}$ at some azimuth $\theta$ of the ring, where $j$ indicates how often the bunch has traveled around the ring. Since each initial phase space position leads to a different particle trajectory with different spin motion, spin fields in general change from turn to turn. A special spin field that does not change from turn to turn is called the invariant spin field $\vec{n}(\vec{z})$ [3].

When a beam is polarized according to this spin field, $\vec{f}(\vec{z}, j)=\vec{n}(\vec{z})$, then the beam polarization is given by the 
TABLE I. Five standard choices of snake schemes. The right column shows an obvious notation to describe the snake angles in a snake scheme.

\begin{tabular}{lcccc}
\hline \hline Scheme & South & East & North & West \\
\hline$\left(0 \frac{\pi}{2} 00\right)$ & $0^{\circ}$ & $90^{\circ}$ & $0^{\circ}$ & $0^{\circ}$ \\
$\left(\frac{\pi}{4} 0 \frac{\pi}{4} 0\right)$ & $45^{\circ}$ & $0^{\circ}$ & $45^{\circ}$ & $0^{\circ}$ \\
$\left(\frac{3 \pi}{4} 0 \frac{3 \pi}{4} 0\right)$ & $-45^{\circ}$ & $0^{\circ}$ & $-45^{\circ}$ & $0^{\circ}$ \\
$\left(\frac{5 \pi}{8} \frac{\pi}{8} \frac{5 \pi}{8} \frac{\pi}{8}\right)$ & $-22.5^{\circ}$ & $22.5^{\circ}$ & $-22.5^{\circ}$ & $22.5^{\circ}$ \\
$\left(\frac{\pi}{8} \frac{5 \pi}{8} \frac{\pi}{8} \frac{5 \pi}{8}\right)$ & $22.5^{\circ}$ & $-22.5^{\circ}$ & $22.5^{\circ}$ & $-22.5^{\circ}$ \\
\hline \hline
\end{tabular}

phase space average $P_{\lim }=\langle\vec{n}(\vec{z})\rangle_{\vec{z}}$. In any case, even when a particle at $\vec{z}(j)$ after its $j$ th turns has a classical spin vector $\vec{S}$ with a direction which is not parallel to $\vec{n}(\vec{z}(j))$, its projection onto the invariant spin field is constant, i.e., $\vec{f}(\vec{z}(j), j) \cdot \vec{n}(\vec{z}(j))$ does not change with $j$. This is possible since its spin $\vec{S}$ appears to rotate around $\vec{n}(\vec{z}(j))$. The time averaged polarization at the phase space point $\vec{z}$ is therefore parallel to $\vec{n}(\vec{z})$ for any spin field. The maximum time averaged polarization at that point is thus given when the beam is initially polarized parallel to $\vec{n}(\vec{z})$. The average $P_{\lim }$ is therefore called the limiting polarization [4-6].

To illustrate one of the benefits of the proposed choice of Siberian snakes it will be shown that they increase $P_{\lim }$.

When an invariant spin field is found, the rotation of $\vec{S}$ around it can be described by an amplitude-dependent spin tune $\nu(\vec{J})$ that depends on the orbital amplitudes $\vec{J}$ but not on the orbital phase variables. This is important since $\nu(\vec{J})$ does not change from turn to turn and can therefore be used to describe long term coherence with the frequencies of orbital motion. When this amplitude-dependent spin tune is in resonance with the orbital tunes $Q_{j}$, i.e., $\nu(\vec{J})=k_{0}+\sum_{j} k_{j} Q_{j}$ for integers $k_{j}$, the spin motion can be strongly disturbed.

As another illustration of the benefits of the proposed choice of Siberian snakes it will be shown that they increase the orbital amplitudes for which low order resonance conditions occur.

It can be shown that the projection of the polarization onto the invariant spin field $J_{S}=\vec{S} \cdot \vec{n}(\vec{z})$ is not only invariant in a storage ring, but that it is an adiabatic invariant [7] when parameters of the accelerator, like the beam's energy, change. This means that a beam that is initially polarized parallel to the invariant spin field $\vec{n}\left(\vec{z}, E_{i}\right)$ and thus has the average polarization $P_{\lim }\left(E_{i}\right)$ at its initial energy $E_{i}$ will still be polarized parallel to the invariant spin field $\vec{n}\left(\vec{z}, E_{f}\right)$ after the beam has been accelerated to a final energy $E_{f}$, and its average polarization is $P_{\text {lim }}\left(E_{f}\right)$. This final polarization can be very large, even though it may have been very small at some intermediate energies. All this is true only if the change of energy is performed adiabatically slowly. Usually, the acceleration cannot be performed adiabatically slowly at all energies and polarization is lost, i.e., the final polarization is smaller than $P_{\lim }\left(E_{f}\right)$.

The most convincing illustration of the benefits of the proposed choice of Siberian snakes is finally that they increase the polarization that is retained after acceleration.

Although it has been straightforward to define $\vec{n}(\vec{z})$, it is not easy to calculate this spin field in general and much effort has been spent on this topic, mostly for electrons at energies up to $46 \mathrm{GeV}$. All algorithms developed before the polarized proton project at HERA- $p$ rely on perturbation methods at some stage, and either do not go to high enough order [8,9] or have problems with convergence at high order and high proton energies [10-12]. The algorithms developed for the HERA- $p$ project [4,5,13-15] made the here presented analysis possible.

\section{MOTIVATION FOR THE OPTIMIZATION OF SIBERIAN SNAKES}

Siberian snakes are indispensable if polarized proton beams are to be accelerated in a high-energy synchrotron. This has the following reasons.

(i) Siberian snakes fix the design orbit spin tune $\nu_{0}$ to $\frac{1}{2}$ during the acceleration cycle so that no first-order resonances have to be crossed. Crossing first-order resonances can lead to a severe reduction of polarization by an amount described by the Froissart-Stora formula [16].

(ii) Siberian snakes strongly reduce the influence of energy variations on spin motion within a synchrotron period [17].

(iii) Siberian snakes reduce the variation of $\vec{n}(\vec{z})$ for particles which oscillate vertically and therefore pass through horizontal fields which perturb the spin motion.

(iv) When $\vec{n}$ changes rapidly during acceleration, the adiabatic invariance of $J_{S}=\vec{n}(\vec{z}) \cdot \vec{S}$ might be violated and polarization would be reduced. It is therefore important that Siberian snakes smooth the changes of $\vec{n}$ during the acceleration cycle.

(v) The reduced variation of $\vec{n}$ is often accompanied by a reduced variation of the amplitude dependent spin tune $\nu$ with energy. This is essential to avoid the crossing of higher-order resonances.

(vi) Siberian snakes can also compensate perturbing effects of misaligned optical elements [17-19] but the effect of misalignments will not be covered here.

To motivate the optimization of the number of Siberian snakes and of their snake axes to which the subsequent sections are dedicated, it will here be shown that different snake arrangements can have quite different quality in respect to the above mentioned reasons.

Useful directions for the snake axes are either vertical or in the horizontal plane. When the snake axis is in the horizontal plane, its angle to the radial direction of the ring is referred to as the snake angle. 


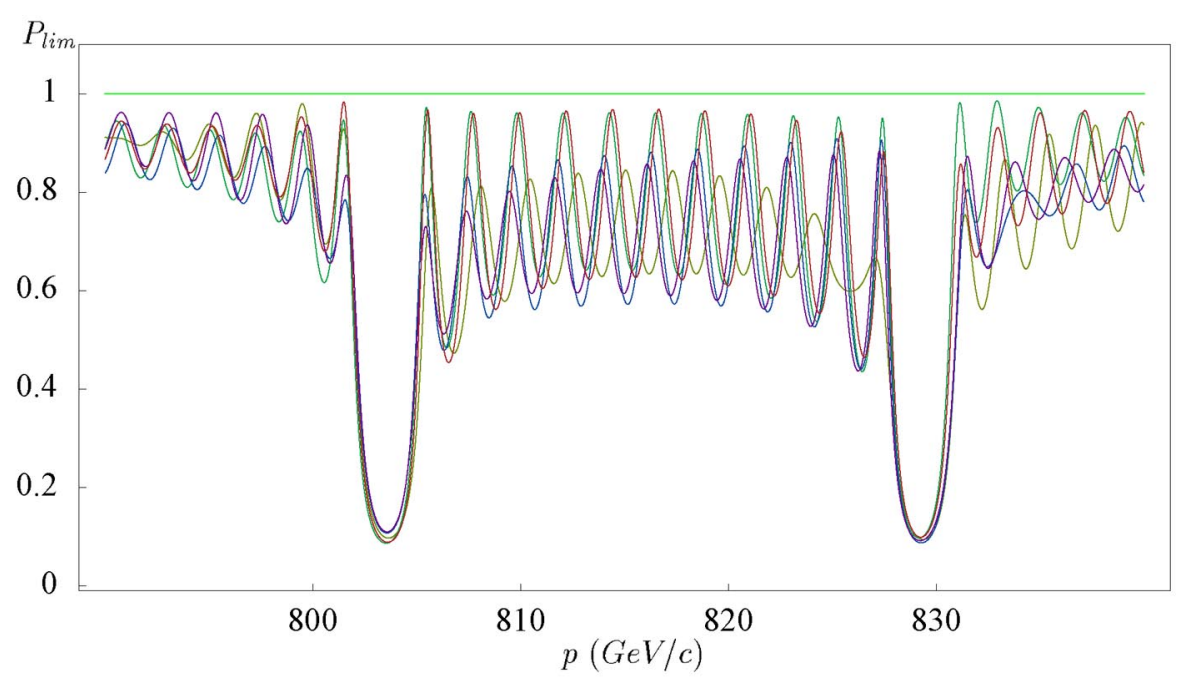

FIG. 1. (Color) $P_{\text {lim }}$ of linearized spin-orbit motion for a $2.5 \sigma$ vertical amplitude for four Siberian snakes in HERA- $p$. Each curve corresponds to one of the five standard choices of snake schemes in Table I.

There is so far no reliable formula for determining the number of Siberian snakes required for an accelerator [20,21]. To make things worse, for any given number of Siberian snakes there are very many different possible combinations of the snake angles which lead to an energy independent closed-orbit spin tune of $\frac{1}{2}$ and to a vertical design orbit spin direction $\vec{n}_{0}$ in the accelerator's arcs. But so far there has been no reliable formula for determining which of these snake schemes leads to the highest polarization.

There used to be a popular opinion that, owing to their symmetry, five standard choices of the snake angles for four Siberian snakes are advantageous for HERA- $p$. For other accelerators similar simplistic choices have been propagated. These choices are not optimal, as will be shown. For reasons that these standard schemes were considered useful see, for example, [22]. RHIC with its two snakes is operated with a similar standard scheme [23].

The energy dependence of $P_{\lim }$ in HERA- $p$ produced by these five snake schemes is shown in Fig. 1. They seem to produce rather similar but very low maximum time average polarization $P_{\lim }$ in a critical energy region where very strong resonances are excited. The observation of such rather small differences in the $\vec{n}$ axis for such different schemes might suggest that all snake arrangements lead to similar $P_{\text {lim }}$, but this is far from true.

Figure 2 (left) shows $P_{\text {lim }}$ as computed for linearized spin-orbit motion [4] for four other schemes with four Siberian snakes which were found by a numerical search for suitable snake angles [4,24]. It is apparent that large increases in $P_{\text {lim }}$ can result from the choice of a suitable snake scheme.

All numerical examples in this paper have been computed for HERA- $p$, the proton ring of an electron-proton collider in Hamburg. The current optics has been used and the only modification was that some magnetic fields have been added in the nonflat sections of HERA- $p$ to simplify the spin dynamics, so that $\vec{n}_{0}$ is vertical in the arcs. The details of these fields can be found $[25,26]$. As a $1 \sigma$ emittance a realistic value of $4 \pi \mathrm{mm} \mathrm{mrad}$ was assumed throughout this paper.

\section{SPIN-ORBIT-COUPLING INTEGRALS}

For the spin-orbit-coupling integrals in flat rings of Eq. (1), $\vec{n}_{0}$ was assumed to point vertically upward. Now Siberian snakes will be included, which rotate all spins
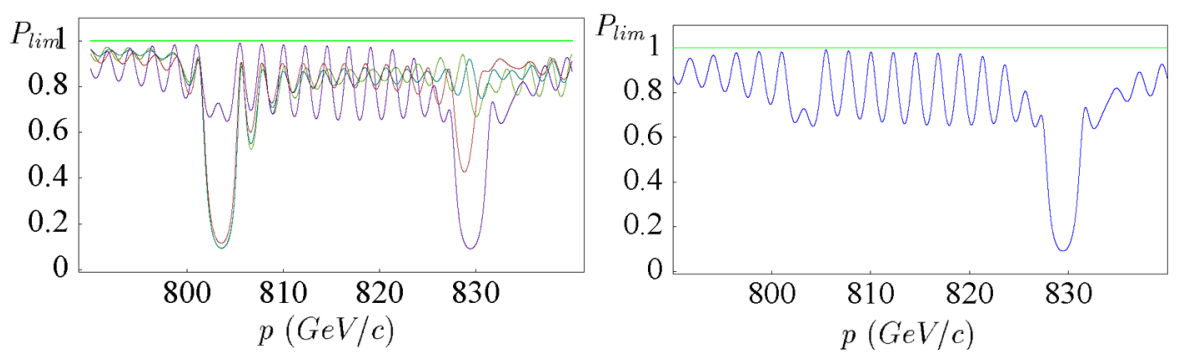

FIG. 2. (Color) $P_{\text {lim }}$ for four different snake schemes which lead to very different maximum time average polarization for the standard HERA- $p$ lattice. Right: one of these snake schemes which were found by numerical optimization of the snake angles. 
and also $\vec{n}_{0}$ by $\pi$ around some axis in the horizontal plane, so that $\vec{n}_{0}$ points downward in some sections. This changes the sign of $\Psi$ in Eq. (1).

It is assumed that there are $n$ Siberian snakes in the ring and that $n$ is even, to make $\vec{n}_{0}$ vertical in the arcs of the ring. The azimuth at the position of a snake is denoted by $\theta_{j}$ and the spin phase advance around the vertically upward direction between snake $j$ and $j+1$ is denoted by $\Psi_{j}$. The spin phase advance after the $j$ th Siberian snake is $\Psi_{j}(\theta)$ with $\Psi_{j}\left(\theta_{j}\right)=0$.

For simplicity $\theta_{0}=0$ and $\theta_{n+1}=2 \pi$ is used and the spin phase advance from azimuth $\theta_{0}$ to the first Siberian snake is $\Psi_{0}$.

In the following a Siberian snake with a snake axis which is in the horizontal plane will be referred to as a horizontal Siberian snake, and for historical reasons a snake which rotates spins around the vertical by some rotation angle will be referred to as a type III snake. A horizontal Siberian snake with a snake angle $\varphi$ is equivalent to a radial Siberian snake followed by a type III snake that introduces an extra spin phase advance of $\alpha=$ $2 \varphi[27]$.

With these notations the spin-orbit-coupling integrals for a ring with horizontal Siberian snakes are

$I_{y}^{ \pm}=\sum_{j=0}^{n} e^{-i \sum_{k=0}^{j-1}(-)^{k}\left(\alpha_{k}+\Psi_{k}\right)} \int_{\theta_{j}}^{\theta_{j+1}} k_{y} e^{i\left[-(-)^{j}\left(\Psi_{j}(\theta)+\alpha_{j}\right) \pm \Phi\right]} d \theta$.

In terms of the orbital phase advance $\Phi_{j}$ between snake $j$ and $j+1$, one obtains

$$
\begin{aligned}
I_{y}^{ \pm}= & \sum_{j=0}^{n} e^{i \sum_{k=0}^{j-1}\left[-(-)^{k}\left(\alpha_{k}+\Psi_{k}\right) \pm \Phi_{k}\right]} \\
& \times \int_{\theta_{j}}^{\theta_{j+1}} k_{y} e^{i\left[-(-)^{j}\left(\Psi_{j}(\theta)+\alpha_{j}\right) \pm \Phi_{j}(\theta)\right]} d \theta .
\end{aligned}
$$

Note that the notation $\sum_{k=0}^{-1}=0$ has been used, and $\alpha_{0}=0$.

A corresponding formula has been used in [1] to introduce so-called strong spin matching, where Siberian snakes are used to produce a cancellation of spin perturbations in different FODO cells.

The spin phase advances between snakes must satisfy the condition $\sum_{k=0}^{n}(-)^{k} \Psi_{k}=0$ to make the closed-orbit spin tune independent of energy and the snake angles must satisfy the condition $\sum_{k=1}^{n}(-)^{k} \varphi_{k}=\frac{\pi}{2} \bmod \pi$ to make the closed-orbit spin tune $\nu_{0}$ equal to $\frac{1}{2}$.

For simplicity we place the last snake at $\theta_{n}=2 \pi$, so that $\alpha_{n}$ does not appear. This simplification is possible since $I^{ \pm}$is an integral around the ring and its amplitude does not depend on where the integration starts. We will try to find optimized $\alpha_{n}$ to make $I^{+}$as well as $I^{-}$zero for all energies. Nevertheless, the fact that $\alpha_{n}$ does not appear does not reduce the number of optimization parameters since $\alpha_{n}$ is no free parameter since the closed-orbit spin tune has to be $\frac{1}{2}$.

\section{SNAKE MATCHING IN RINGS WITH SUPERPERIODICITY}

The spin perturbations in different parts of the ring can compensate each other when these parts have similar spin-orbit-coupling integrals. This is achieved by using the Siberian snakes to adjust the spin phase advances in such a way that spin-orbit-coupling integrals of similar parts of a ring cancel each other. In the following, the process of finding a snake scheme for which such a compensation occurs will be referred to as snake matching [28,29]. After demonstrating the idea for type III snakes, which simply rotate spins around the vertical by some fixed angle with little influence on the orbit motion, three quite general results will be demonstrated.

(1) A ring with superperiodicity 4 can be completely snake matched using eight Siberian snakes, i.e., a snake scheme can be found for which the spin-orbit-coupling integrals are zero due to a complete cancellation of spin perturbations in different parts of the ring. There are exactly two such possibilities which lead to energy independent snake angles.

(2) Such a ring can also be snake matched using four Siberian snakes. Then, however, the snake angles depend on energy and have to be changed during the acceleration process.

(3) Energy independent snake matching with four Siberian snakes can be found when the betatron phase advance is appropriately chosen for each of the four quadrants.

\section{A. Snake matching with type III snakes for super- periodicity 4}

In this section the benefit of type III snakes will be analyzed, there are no radial Siberian snakes in the ring here. The index $y$ on the spin-orbit-coupling integral and on the vertical phase advance and tune will not be indicated. In any case, the methods for canceling spin-orbitcoupling integrals by a special choice of snake angles which will now be derived can also be used for transverse and longitudinal motion. In this section the notation will be further simplified by using the symbols $\nu_{0}$ and $Q$ to denote $2 \pi$ times the spin tune and $2 \pi$ times the orbital tune. Then for a ring with superperiodicity $4, I^{ \pm}$can be computed from

$$
\begin{aligned}
I_{1 / 4}^{ \pm}= & \int_{0}^{\pi / 2} k_{y} e^{i[-\Psi(\theta) \pm \Phi(\theta)]} d \theta, \\
I^{ \pm}= & I_{1 / 4}^{ \pm}\left[1+e^{i\left(-\nu_{0} \pm Q\right) / 4}+e^{i 2\left(-\nu_{0} \pm Q\right) / 4}\right. \\
& \left.+e^{i 3\left(-\nu_{0} \pm Q\right) / 4}\right] .
\end{aligned}
$$

Spin transparency requires that $I^{+}$as well as $I^{-}$vanish. 


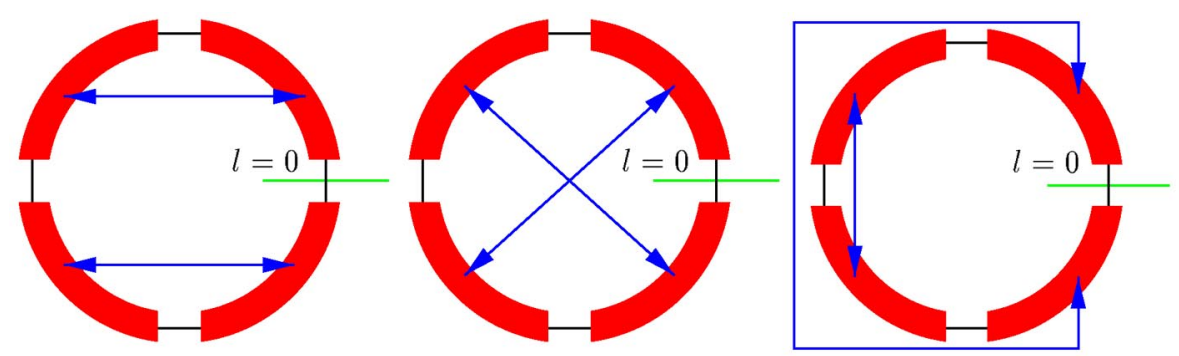

FIG. 3. (Color) The three possibilities for canceling the depolarizing effects of quadrants of a ring with superperiodicity 4 . The arrows indicate which quadrants cancel.

Thus the bracket in (4) must vanish. This is only possible when $e^{i\left(-\nu_{0} \pm Q\right) / 4}$ is either -1 or $i$. Choosing the first possibility to eliminate $I^{+}$and the second to eliminate $I^{-}$, one obtains

$$
e^{i\left(-\nu_{0}+Q\right) / 4}=-1, \quad e^{i\left(-\nu_{0}-Q\right) / 4}=i .
$$

This leads to the requirement $e^{i Q / 2}=i$ which cannot be satisfied in a realistic ring. Therefore, a fourfold repetitive symmetry cannot lead to spin transparency at any energy. While the spin disturbance of two quadrants can therefore not cancel in $I^{+}$as well as in $I^{-}$, one of these integrals can cancel whenever the spin phase advance between the quadrants is appropriate.

The situation changes if type III snakes are installed. As first found in [24], type III snakes can improve the spin dynamics in HERA- $p$ by increasing $P_{\text {lim }}=|\langle\vec{n}\rangle|$. They can be used to manipulate the spin phase advance to make the spin-orbit-coupling integrals of different parts of the ring cancel. To demonstrate this, four type III snakes are installed regularly spaced around the ring.

There are three possibilities for canceling the spin disturbances between quadrants of the ring. The quadrants whose destructive effects cancel are connected by arrows in Fig. 3.

The spin-orbit-coupling integrals are

$$
\begin{aligned}
I^{ \pm}= & I_{1 / 4}^{ \pm}\left[1+e^{i\left(-\nu_{0} \pm Q\right) / 4-\psi_{1}}+e^{i 2\left(-\nu_{0} \pm Q\right) / 4-\psi_{1}-\psi_{2}}\right. \\
& \left.+e^{i 3\left(-\nu_{0} \pm Q\right) / 4-\psi_{1}-\psi_{2}-\psi_{3}}\right]
\end{aligned}
$$

where $\psi_{j}$ is the spin rotation angle of the type III snake at $\theta=j \frac{\pi}{2}$. To snake match the ring, $I^{+}$as well as $I^{-}$must vanish. Therefore the bracket on the right-hand side has to vanish in both cases. A sum of four complex numbers with unit modulus can only vanish when it consist of two pairs of numbers which cancel each other. This is shown in Fig. 4.

The three possibilities of cancellation demonstrated in Fig. 3 are given by the following three sets of equations:

$$
\begin{array}{lr}
\text { 1. } & \left(-\nu_{0} \pm Q\right) / 4-\psi_{1} \stackrel{\circ}{=} \pi \\
\text { and } & \left(-\nu_{0} \pm Q\right) / 4-\psi_{3} \stackrel{\circ}{=} \pi \\
\text { 2. } & 2\left(-\nu_{0} \pm Q\right) / 4-\psi_{1}-\psi_{2} \stackrel{\circ}{=} \pi \\
\text { and } & \psi_{3} \stackrel{\circ}{=} \psi_{1} \\
\text { 3. } & 3\left(-\nu_{0} \pm Q\right) / 4-\psi_{1}-\psi_{2}-\psi_{3} \stackrel{\circ}{=} \pi \\
\text { and } & \left(-\nu_{0} \pm Q\right) / 4-\psi_{2} \stackrel{\circ}{=} \pi
\end{array}
$$

The symbol $\stackrel{\circ}{=}$ indicates equivalence modulo $2 \pi$. To snake match, one of these three conditions has to hold for $\left(-\nu_{0}+Q\right)$, which lets $I^{+}$vanish and another of the conditions has to hold for $\left(-\nu_{0}-Q\right)$, which lets $I^{-}$ vanish. $I^{+}$and $I^{-}$cannot vanish due to the same condition if restrictions on the allowed orbital phase advance $Q$ are to be avoided. There are therefore three possibilities.

(i) $I^{+}=0$ due to condition 2 and $I^{-}=0$ due to condition 3 requires

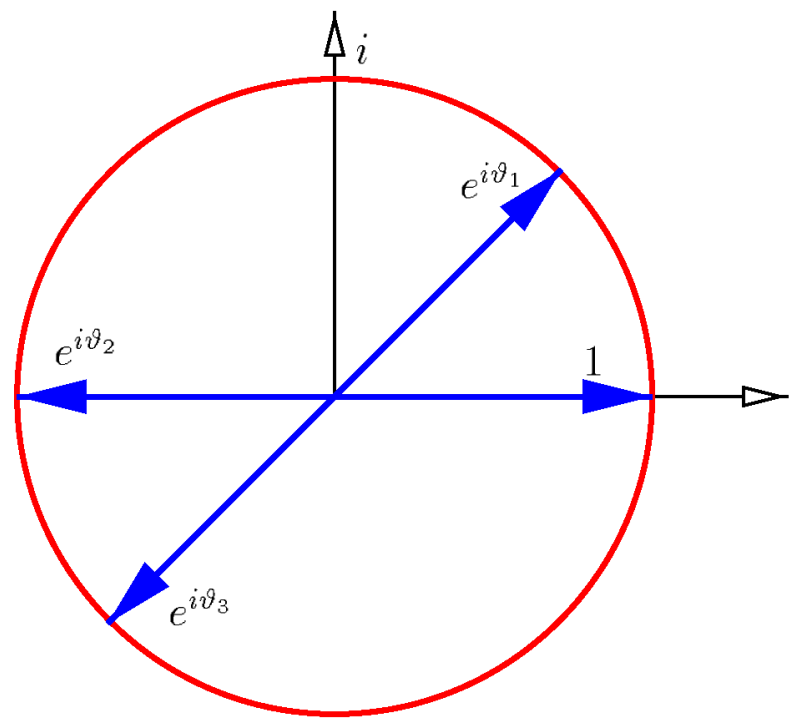

FIG. 4. (Color) Four complex numbers with modulus 1 can only add up to zero when they consist of two pairs which individually add up to zero. 


$$
\begin{aligned}
& \psi_{2} \stackrel{\circ}{=} \pi+2\left(-\nu_{0}+Q\right) / 4-\psi_{1}, \quad \psi_{3} \stackrel{\circ}{=} \psi_{1}, \\
& \psi_{2} \stackrel{\circ}{=} \pi+\left(-\nu_{0}-Q\right) / 4, \quad \psi_{3} \stackrel{\circ}{=} 2\left(-\nu_{0}-Q\right) / 4-\psi_{1} .
\end{aligned}
$$

The first and third of these equations require that $\psi_{1} \stackrel{\circ}{=}\left(-\nu_{0}+3 Q\right) / 4$, whereas the second and fourth equations require that $\psi_{1} \stackrel{\circ}{=}\left(-\nu_{0}-Q\right) / 4$. These two requirements are in general not compatible and the ring cannot be made spin transparent in this way.

(ii) $I^{+}=0$ due to condition 1 and $I^{-}=0$ due to condition 3 requires

$$
\begin{array}{ll}
\psi_{1} \stackrel{\circ}{=} \pi+\left(-\nu_{0}+Q\right) / 4, & \psi_{3} \stackrel{\circ}{=} \psi_{1}, \\
\psi_{2} \stackrel{\circ}{=} \pi+\left(-\nu_{0}-Q\right) / 4, & \psi_{3} \stackrel{\circ}{=} 2\left(-\nu_{0}-Q\right) / 4-\psi_{1} .
\end{array}
$$

The first and the last of these equations together require $\psi_{3} \stackrel{\circ}{=} \pi-\left(3 Q-\nu_{0}\right) / 4$. This is in conflict with the second equation. Thus this way also cannot lead to a spin transparent ring.

(iii) $I^{+}=0$ due to condition 1 and $I^{-}=0$ due to condition 2 requires

$$
\begin{aligned}
& \psi_{1} \stackrel{\circ}{=} \pi+\left(-\nu_{0}+Q\right) / 4, \quad \psi_{3} \stackrel{\circ}{=} \psi_{1}, \\
& \psi_{2} \stackrel{\circ}{=} \pi+2\left(-\nu_{0}-Q\right) / 4-\psi_{1}, \quad \psi_{3} \stackrel{\circ}{=} \psi_{1} .
\end{aligned}
$$

These four equations are compatible and lead to $\psi_{1} \stackrel{\circ}{=} \psi_{3} \stackrel{\circ}{=} \pi+\left(-\nu_{0}+Q\right) / 4$ and $\psi_{2} \stackrel{\circ}{=}\left(-\nu_{0}-3 Q\right) / 4$.

The type III snake at $l=0$ has the rotation angle $\psi_{4}$, which is chosen in such a way that the closed-orbit spin tune of the ring does not change due to the snakes, i.e., $\psi_{1}+\psi_{2}+\psi_{3}+\psi_{4} \stackrel{\circ}{=} 0$. The required rotation angles are then

$$
\begin{aligned}
& \psi_{1} \stackrel{\circ}{=} \psi_{3} \stackrel{\circ}{=} \pi+\frac{-\nu_{0}+Q}{4}, \quad \psi_{2} \stackrel{\circ}{=}-\frac{\nu_{0}+3 Q}{4}, \\
& \psi_{4} \stackrel{\circ}{=} \frac{Q-3-\nu_{0}}{4} .
\end{aligned}
$$

Obviously a change in sign of $Q$ leads to $I^{+}=0$ due to condition 2 and to $I^{-}=0$ due to condition 1 . There are therefore exactly two possibilities for making a ring with superperiodicity 4 spin transparent by means of four type III snakes. These possibilities are shown in Fig. 5.

However, the scheme of four type III snakes presented here cannot be a practical snake scheme, since it does not make the closed-orbit spin tune independent of energy. But it illustrates how type III snakes can be used at fixed energy to make spin perturbations from different parts of the ring cancel each other. This feature can then be used in combination with the Siberian snakes which are installed to make the closed-orbit spin tune independent of energy.

\section{B. Snake matching with type III snakes for superper- iodicity 4 and mirror symmetry}

Again, only type III snakes and no radial Siberian snakes are analyzed here. In particle optical systems, mirror symmetries are often used to cancel perturbative effects [30-33]. Therefore it is interesting to see whether mirror symmetry can lead to vanishing of spin-orbitcoupling integrals when four Siberian snakes are installed at the symmetry points of the ring. If one superperiod is mirror symmetric, then

$$
\begin{aligned}
& I_{1 / 8}^{ \pm}=\int_{0}^{\pi / 4} k_{y} e^{i[-\Psi(\theta) \pm \Phi(\theta)]} d \theta, \\
& I_{1 / 4}^{ \pm}=I_{1 / 8}^{ \pm}+\left(I_{1 / 8}^{ \pm}\right)^{*} e^{i\left(-\nu_{0} \pm Q\right) / 4} .
\end{aligned}
$$

With $\Sigma^{ \pm}=\left(-\nu_{0} \pm Q\right) / 4$ one obtains for the complete
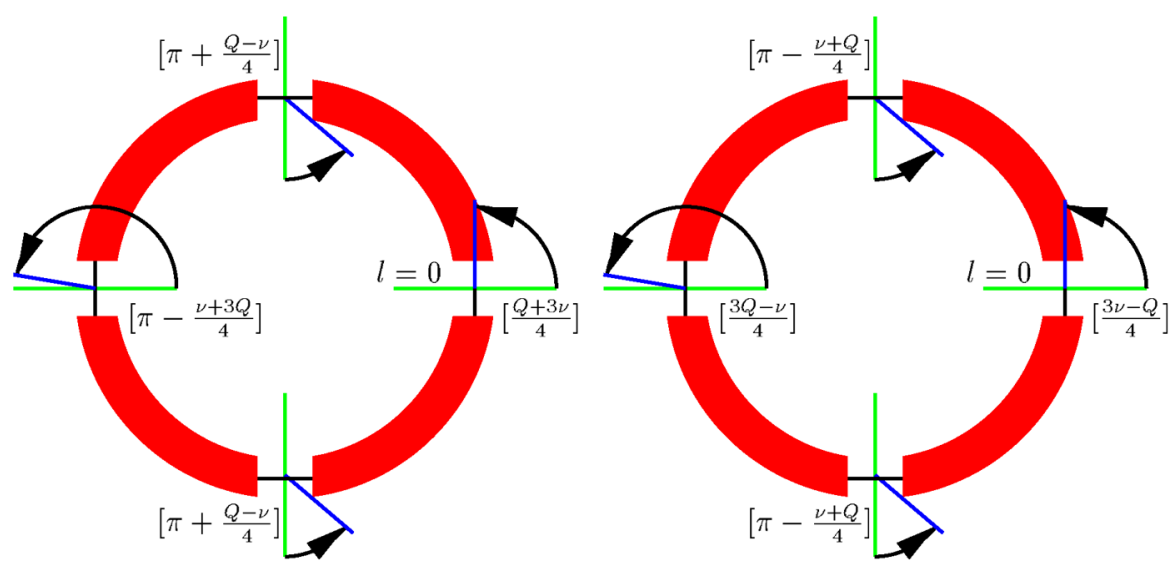

FIG. 5. (Color) The only two ways to snake match a ring with superperiodicity 4 by four type III snakes. The number $[x]$ denotes $x$ mod $2 \pi$ and lies in $[0,2 \pi)$. The vertical tune times $2 \pi$ is denoted by $Q$ and $\nu_{0}=G \gamma 2 \pi$. 
ring

$$
I^{ \pm}=\left[I_{1 / 8}^{ \pm}+\left(I_{1 / 8}^{ \pm}\right)^{*} e^{i \Sigma^{ \pm}}\right] \cdot\left(1+e^{i \Sigma^{ \pm}}+e^{i 2 \Sigma^{ \pm}}+e^{i 3 \Sigma^{ \pm}}\right) .
$$

Thus, snake matching the ring with superperiodicity 4 is not influenced by the fact that the ring might have a mirror symmetry since the bracket in (14) is equivalent to the corresponding bracket in (4) for rings without mirror symmetry.

Type III snakes will not be considered further, since a horizontal Siberian snake with snake angle $\varphi$ can be decomposed into a radial Siberian snake and a type III snake with rotation angle $2 \varphi$.

\section{Snake matching of Siberian snakes with fixed axes at all energies for superperiodicity 4}

\section{Schemes with four snakes}

For four horizontal Siberian snakes the spin-orbitcoupling integral in (3) is

$$
\begin{aligned}
I^{ \pm}= & \int_{\theta_{0}}^{\theta_{1}} k_{y} e^{i\left[-\Psi_{0}(\theta) \pm \Phi_{0}(\theta)\right]} d \theta+e^{i\left(-\Psi_{0} \pm \Phi_{0}\right)} \\
& \times \int_{\theta_{1}}^{\theta_{2}} k_{y} e^{i\left[\Psi_{1}(\theta)+\alpha_{1} \pm \Phi_{1}(\theta)\right]} d \theta+e^{i\left[-\Psi_{0}+\alpha_{1}+\Psi_{1} \pm\left(\Phi_{0}+\Phi_{1}\right)\right]} \\
& \times \int_{\theta_{2}}^{\theta_{3}} k_{y} e^{i\left[-\Psi_{2}(\theta)-\alpha_{2} \pm \Phi_{2}(\theta)\right]} d \theta \\
& +e^{i\left[-\Psi_{0}+\alpha_{1}+\Psi_{1}-\alpha_{2}-\Psi_{2} \pm\left(\Phi_{0}+\Phi_{1}+\Phi_{2}\right)\right]} \\
& \times \int_{\theta_{3}}^{2 \pi} k_{y} e^{i\left[\Psi_{3}(\theta)+\alpha_{3} \pm \Phi_{3}(\theta)\right]} d \theta
\end{aligned}
$$

For a ring with superperiodicity 4 and with four equally spaced horizontal Siberian snakes one obtains with $\nu_{0}=$ $\Psi(2 \pi), \Psi_{j}=\Psi(2 \pi) / 4$, and $\Phi_{j}=Q / 4$ the relation

$$
\begin{gathered}
I_{1 / 4}^{ \pm}=\int_{0}^{\pi / 2} k_{y} e^{i[-\Psi(\theta) \pm \Phi(\theta)]} d \theta \\
I^{ \pm}=I_{1 / 4}^{ \pm}\left(1+e^{i\left[-\Psi_{0}+\alpha_{1}+\Psi_{1}-\alpha_{2} \pm\left(\Phi_{0}+\Phi_{1}\right)\right]}\right) \\
+\left(I_{1 / 4}^{\mp}\right)^{*} e^{i\left(-\Psi_{0}+\alpha_{1} \pm \Phi_{0}\right)}\left(1+e^{i\left[\Psi_{1}-\alpha_{2}-\Psi_{2}+\alpha_{3} \pm\left(\Phi_{1}+\Phi_{2}\right)\right]}\right) \\
=I_{1 / 4}^{ \pm}\left(1+e^{i\left(\alpha_{1}-\alpha_{2} \pm 2 Q / 4\right)}\right)+\left(I_{1 / 4}^{\mp}\right)^{*} e^{i\left[\left(-\nu_{0} \pm Q\right) / 4+\alpha_{1}\right]}(1 \\
\left.+e^{i\left(-\alpha_{2}+\alpha_{3} \pm 2 Q / 4\right)}\right) .
\end{gathered}
$$

Spin transparency of the ring is therefore obtained when $\alpha_{1}-\alpha_{2} \pm 2 Q / 4 \stackrel{\circ}{=} \pi \quad$ and $\quad-\alpha_{2}+\alpha_{3} \pm 2 Q / 4 \stackrel{\circ}{=} \pi$.

This cannot be achieved in general since the conditions $\alpha_{1}-\alpha_{2}+2 Q / 4 \stackrel{\circ}{=} \pi$ and $\alpha_{1}-\alpha_{2}-2 Q / 4 \stackrel{\circ}{=} \pi$ have to be satisfied simultaneously, which implies $Q \stackrel{\circ}{=} 0$.

In the case of mirror symmetry in the ring $I_{1 / 4}^{+}$and $I_{1 / 4}^{-}$ are related by (13)

$$
\begin{gathered}
I_{1 / 4}^{ \pm}=I_{1 / 8}^{ \pm}+\left(I_{1 / 8}^{ \pm}\right)^{*} e^{i\left(-\nu_{0} \pm Q\right) / 4} \\
\left(I_{1 / 4}^{\mp}\right)^{*}=\left(I_{1 / 8}^{\mp}\right)^{*}+I_{1 / 8}^{\mp} e^{-i\left(-\nu_{0} \mp Q\right) / 4}=I_{1 / 4}^{\mp} e^{-i\left(-\nu_{0} \mp Q\right) / 4} .
\end{gathered}
$$

With mirror symmetric quadrants the spin-orbitcoupling integral then simplifies to

$$
\begin{aligned}
I^{ \pm}= & I_{1 / 4}^{ \pm}\left(1+e^{i\left(\alpha_{1}-\alpha_{2} \pm 2 Q / 4\right)}\right)+I_{1 / 4}^{\mp}(1 \\
& \left.+e^{i\left(-\alpha_{2}+\alpha_{3} \pm 2 Q / 4\right)}\right) e^{i\left(\alpha_{1} \pm 2 Q / 4\right)},
\end{aligned}
$$

and again this additional symmetry does not simplify the compensation of the spin-orbit integrals.

\section{Schemes with eight snakes}

The same procedure can now be repeated with eight snakes. For that purpose four more horizontal Siberian snakes are placed at the locations $j \pi / 2+\Delta \theta, j \in$ $\{0,1,2,3\}$. In terms of the integrals

$$
\begin{gathered}
I_{0}^{ \pm}=\int_{0}^{\Delta \theta} k_{y} e^{i[-\Psi(\theta) \pm \Phi(\theta)]} d \theta, \\
I_{1}^{ \pm}=\int_{\Delta \theta}^{\pi / 2} k_{y} e^{i[\Psi(\theta) \pm \Phi(\theta)]} d \theta,
\end{gathered}
$$

the spin-orbit-coupling integrals of (3) are

$$
\begin{aligned}
I^{ \pm}= & I_{0}^{ \pm}\left(1+e^{i\left[-\Psi_{0}+\alpha_{1}+\Psi_{1}-\alpha_{2} \pm\left(\Phi_{0}+\Phi_{1}\right)\right]}+\cdots\right. \\
& \left.+e^{i\left[-\Psi_{0}+\alpha_{1}+\Psi_{1}-\alpha_{2} \mp \cdots-\alpha_{n-2} \pm\left(\Phi_{0}+\cdots+\Phi_{n-3}\right)\right]}\right) \\
& +I_{1}^{ \pm} e^{i\left(-\Psi_{0}+\alpha_{1} \pm \Phi_{0}\right)}\left(1+e^{i\left[\Psi_{1}-\alpha_{2}-\Psi_{2}+\alpha_{3} \pm\left(\Phi_{1}+\Phi_{2}\right)\right]}\right. \\
& \left.+\cdots+e^{i\left[\Psi_{1}-\alpha_{2}-\Psi_{2}+\alpha_{3} \pm \cdots+\alpha_{n-1} \pm\left(\Phi_{1}+\cdots+\Phi_{n-2}\right)\right]}\right) .
\end{aligned}
$$

If there is an additional mirror symmetry and the snakes are all placed in the symmetry points, (13) implies $I_{1}^{ \pm}=$ $\left(I_{0}^{ \pm}\right)^{*} e^{i\left(-\nu_{0} \pm Q\right) / 4}$, which again does not lead to simplifications. The complete spin phase advance of the ring is $\sum_{j=0}^{n}(-)^{j}\left(\Psi_{j}+\alpha_{j}\right)=\pi$. Since this phase advance is required to be independent of energy, $\sum_{j=0}^{n}(-)^{j} \Psi_{j}$ has to vanish. Because of the superperiodicity this requires $\Psi_{0}=\Psi_{1}$, and all the spin phases $\Psi_{j}$ in Eq. (25) cancel. Then in terms of the difference angles $\Delta_{j k}=\alpha_{j}-\alpha_{k}$, spin matching the ring requires

$$
\begin{aligned}
1 & +e^{i\left( \pm Q / 4+\Delta_{12}\right)}+e^{i\left( \pm 2 Q / 4+\Delta_{12}+\Delta_{34}\right)} \\
& +e^{i\left( \pm 3 Q / 4+\Delta_{12}+\Delta_{34}+\Delta_{56}\right)} \\
& =0 \\
1 & +e^{i\left( \pm Q / 4-\Delta_{23}\right)}+e^{i\left( \pm 2 Q / 4-\Delta_{23}-\Delta_{45}\right)} \\
& +e^{i\left( \pm 3 Q / 4-\Delta_{23}-\Delta_{45}-\Delta_{67}\right)} \\
& =0 .
\end{aligned}
$$

Sets of four complex numbers with modulus 1 can only add up to zero by the three schemes shown in Fig. 3. 
Equations (25) and (26) have the same structure as the matching conditions of Eq. (6) and the relations (12) can therefore be used to obtain the following two ways to satisfy (25):

$$
\begin{aligned}
\Delta_{12} \stackrel{\circ}{=} \Delta_{56} \stackrel{\circ}{=} \pi-Q / 4, \quad \Delta_{34} \stackrel{\circ}{=} 3 Q / 4, \\
\Delta_{12} \stackrel{\circ}{=} \Delta_{56} \stackrel{\circ}{=} \pi+Q / 4, \quad \Delta_{34} \stackrel{\circ}{=}-3 Q / 4 .
\end{aligned}
$$

Equation (28) resulted from reversing the sign of $Q$ in (27). There are also exactly two possibilities for solving (26):

$$
\begin{aligned}
\Delta_{23} \stackrel{\circ}{=} \Delta_{67} \stackrel{\circ}{=} \pi+Q / 4, \quad \Delta_{45} \stackrel{\circ}{=}-3 Q / 4, \\
\Delta_{23} \stackrel{\circ}{=} \Delta_{67} \stackrel{\circ}{=} \pi-Q / 4, \quad \Delta_{45} \stackrel{\circ}{=} 3 Q / 4 .
\end{aligned}
$$

There are now four possibilities to snake match the ring; these are obtained by combining Eqs. (27) and (29), (27) and (30), (28) and (29), or (28) and (30), where the last two possibilities also result from the first two by reversing the sign of $Q$. Figure 6 shows how parts of the ring cancel the depolarizing effects of other parts in these snake matching schemes.

Since only differences in the snake angles appear, one of the angles can be chosen arbitrarily. This then fixes all other snake angles. Here $\alpha_{1}=0$ is chosen for simplicity. This leads to the following possibilities.

Combination of Eqs. (27) and (29):

$$
\begin{gathered}
\alpha_{1} \stackrel{\circ}{=} 0, \quad \alpha_{2} \stackrel{\circ}{=} \pi+Q / 4, \quad \alpha_{3} \stackrel{\circ}{=} 0, \\
\alpha_{4} \stackrel{\circ}{=}-3 Q / 4, \quad \alpha_{5} \stackrel{\circ}{=} 0, \quad \alpha_{6} \stackrel{\circ}{=} \pi+Q / 4, \\
\alpha_{7} \stackrel{\circ}{=} 0, \quad \alpha_{8} \stackrel{\circ}{=} \pi+Q / 4 .
\end{gathered}
$$

Combination of Eqs. (27) and (30):

$$
\begin{gathered}
\alpha_{1} \stackrel{\circ}{=} 0, \quad \alpha_{2} \stackrel{\circ}{=} \pi+Q / 4, \quad \alpha_{3} \stackrel{\circ}{=} 2 Q / 4, \quad \text { (3) } \\
\alpha_{4} \stackrel{\circ}{=}-Q / 4, \quad \alpha_{5} \stackrel{\circ}{=}-4 Q / 4, \quad \alpha_{6} \stackrel{\circ}{=} \pi-3 Q / 4,
\end{gathered}
$$

$$
\alpha_{7} \stackrel{\circ}{=}-2 Q / 4, \quad \alpha_{8} \stackrel{\circ}{=} \pi-Q / 4 .
$$

The values for $\alpha_{8}$ were obtained from the requirement $\alpha_{8}-\alpha_{7}+\alpha_{6}-\alpha_{5}+\alpha_{4}-\alpha_{3}+\alpha_{2}-\alpha_{1} \stackrel{\circ}{=} \pi$. The last snake scheme can be simplified by decreasing all snake angles by $2 Q / 4$, leading to

$$
\begin{array}{cc}
\alpha_{1} \stackrel{\circ}{=} Q / 4, \quad \alpha_{2} \stackrel{\circ}{=} \pi+2 Q / 4, \quad \alpha_{3} \stackrel{\circ}{=} 3 Q / 4, \\
\alpha_{4} \stackrel{\circ}{=} 0, \quad \alpha_{5} \stackrel{\circ}{=}-3 Q / 4, \quad \alpha_{6} \stackrel{\circ}{=} \pi-2 Q / 4, \\
\alpha_{7} \stackrel{\circ}{=}-Q / 4, \quad \alpha_{8} \stackrel{\circ}{=} \pi .
\end{array}
$$

These snake schemes are shown in Fig. 7 where account has been taken of the fact that the actual angle between the snake's rotation axis and the radial direction is $\alpha / 2$. Furthermore, advantage has been taken of the fact that the angle $\alpha / 2$ only needs to be known modulo $\pi$.

Here it is very important to note that the snake angles are independent of $\nu=G \gamma$ and therefore that a snake match has been achieved for all energies. With four Siberian snakes such an energy independent snake match is not possible in a fourfold symmetric ring.

One could repeat the same procedure for a layout with six horizontal Siberian snakes or with combinations of, for example, six horizontal Siberian snakes and two type III snakes. Because of their threefold symmetry, this could be of special interest for the RHIC rings.
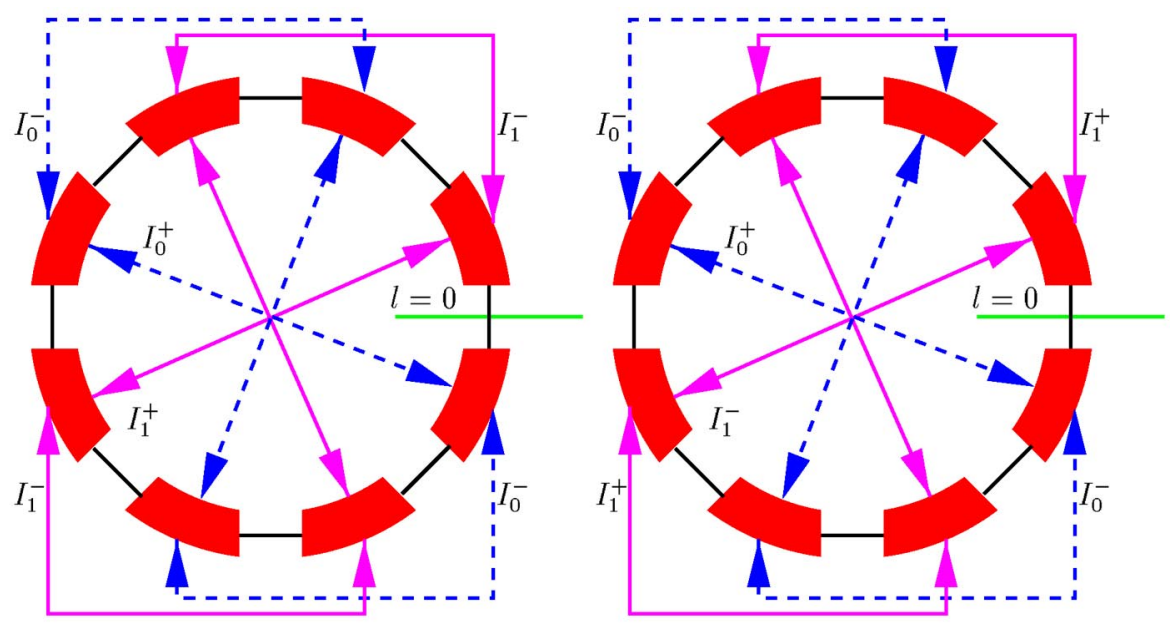

FIG. 6. (Color) The two possibilities by which the individual parts of the ring cancel the spin-orbit-coupling integrals. Changing the sign of $Q$ leads to two corresponding snake schemes. The symbols $I_{0}^{+}, I_{0}^{-}, I_{1}^{+}$, and $I_{1}^{-}$indicate which part of the spin-orbitcoupling integrals are canceled. 


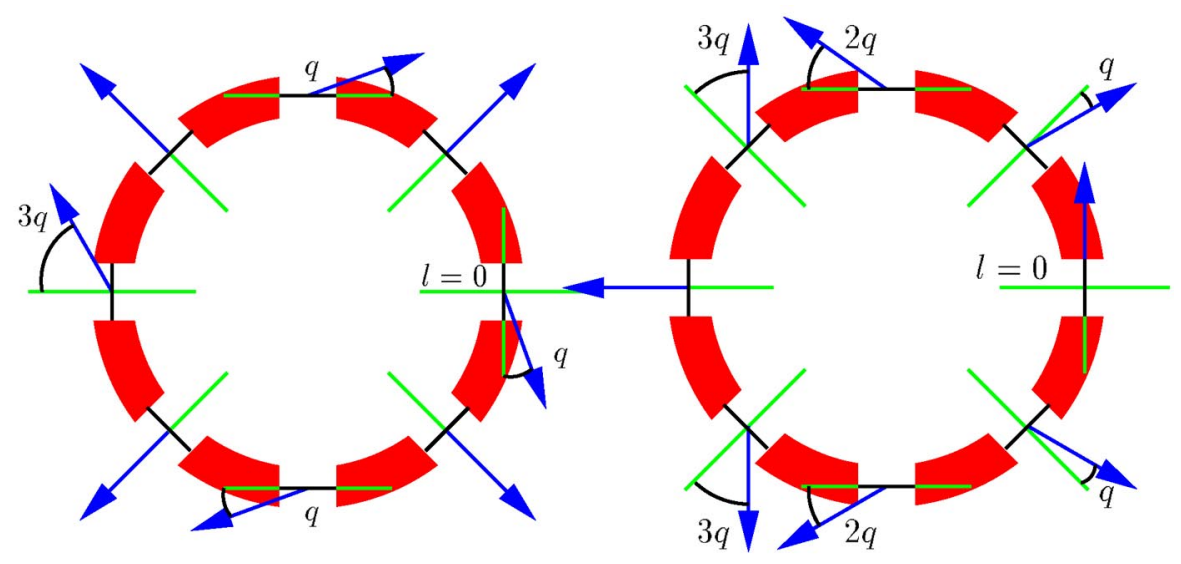

FIG. 7. (Color) Two of the four ways to snake match a ring with superperiodicity 4 using eight horizontal Siberian snakes. The number $q$ denotes $Q / 8 \bmod \pi$ and lies in $[0, \pi)$. The vertical tune times $2 \pi$ is denoted by $Q$ and $\nu=G \gamma 2 \pi$. The other two possible snake schemes are obtained by reversing the sign of $Q$. When all snake angles are increased by the same amount, the ring remains spin transparent. Note that the snake angle is independent of $\nu$ and thus of energy.

\section{SNAKE MATCHING HERA- $p$}

\section{A. Schemes with four snakes}

When the spin-orbit-coupling integrals starting at an azimuth $\theta_{0}$ are minimized, the opening angle of the invariant spin field at $\theta_{0}$ for the approximation of linear spin-orbit motion is also minimized. In fact, $P_{\lim }=1$ in linear approximation if $I^{ \pm}=0$ since then the spin motion is decoupled from the orbit to first order.

It has been seen from the example of a ring with superperiodicity 4 that eight Siberian snakes can be used to snake match the spin-orbit-coupling integrals to zero at one azimuth of the ring for all energies. This could be achieved since the snake angles were used to adjust the spin phase advances in such a way that perturbations in one part of the ring were compensated by identical perturbations in one of the identical superperiods of the ring.

HERA- $p$ has four identical arcs separated by four not identical straight sections. Because of the lack of fourfold symmetry it is in general not possible to find snake angles which completely compensate all spin-orbit-coupling integrals. However, the four identical arc sections of HER A dominate the spin-orbit resonance strength of vertical motion. Thus it would make sense to arrange that the perturbing effect of these arcs cancel each other. A first step in this direction is a symmetrization of the quadrants by making the spin phase advance in all of the straight sections identical. It is possible to add magnetic fields for that purpose [4].

The spin-orbit-coupling integrals from the first regular FODO cell to the last FODO cell of a regular arc in HERA- $p$ will be denoted by $\hat{I}_{y}^{+}$and $\hat{I}_{y}^{-}$and the azimuths of the beginnings of the four regular arcs as $\theta_{1}, \theta_{2}, \theta_{3}$, and $\theta_{4}$. The central points of the south, west, north, and east straight sections are denoted by $S, W, N$, and $E$. The spin phase advances between the arcs are compensated using the snake angles $\varphi_{E}, \varphi_{N}$, and $\varphi_{W}$. The spin phase advance between $\theta_{i}$ and $\theta_{j}$ is denoted by $\Psi_{i j}$. These notations are indicated in Fig. 8.

With Siberian snakes in each of the straight sections, the spin phase advance from $\theta_{1}$ to $\theta_{3}$ is given by $\Psi_{13}=$ $\Psi_{1 E}-2 \varphi_{E}-\Psi_{E N}+2 \varphi_{N}+\Psi_{N 3}$. The spin phase advance is identical in all quadrants of the ring. The total spin phase advance is then solely determined by the snake angles and is therefore independent of energy: $\Psi_{13}=$ $2\left(\varphi_{N}-\varphi_{E}\right)$ and $\Psi_{24}=2\left(\varphi_{W}-\varphi_{N}\right)$. The orbital phase

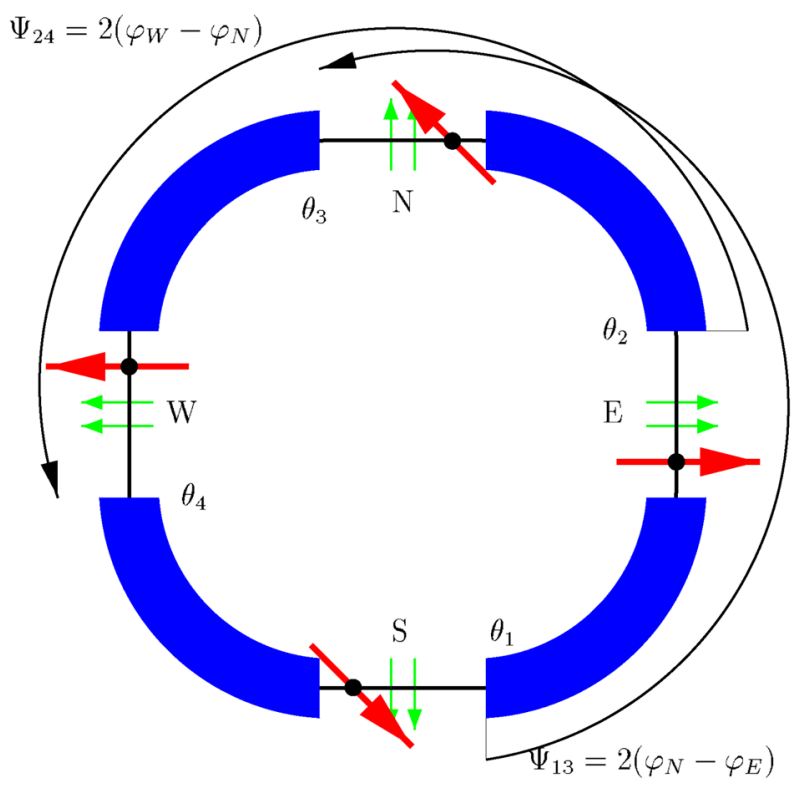

FIG. 8. (Color) The spin phase advance from the beginning of one regular arc to the beginning of the regular arc on the opposite side of the ring. Large arrows indicate Siberian snakes, small arrows indicate the additional magnetic fields which were added to make the spin phase advance in all straight sections identical. 
advance $\Phi\left(\theta_{3}\right)-\Phi\left(\theta_{1}\right)$ also does not depend on energy. For simplicity, $\Phi\left(\theta_{j}\right)-\Phi\left(\theta_{i}\right)$ will now be denoted by $\Phi_{i j}$.

The spin-orbit-coupling integrals at the south interaction point then contain the following contributions from the four regular arcs:

$$
\begin{aligned}
I_{\mathrm{arcs}}^{+}= & \hat{I}^{+} e^{i\left(-\Psi_{S 1}+\Phi_{S 1}\right)}\left(1+e^{i\left[2\left(\varphi_{E}-\varphi_{N}\right)+\Phi_{13}\right]}\right) \\
& +\left(\hat{I}^{-}\right)^{*} e^{i\left(2 \varphi_{E}-\Psi_{S E}+\Psi_{E 2}+\Phi_{S 2}\right)}\left(1+e^{i\left[2\left(\varphi_{W}-\varphi_{N}\right)+\Phi_{24}\right]}\right),
\end{aligned}
$$

$$
\begin{aligned}
I_{\mathrm{arcs}}^{-}= & \hat{I}^{-} e^{i\left(-\Psi_{S 1}-\Phi_{S 1}\right)}\left(1+e^{i\left[2\left(\varphi_{E}-\varphi_{N}\right)-\Phi_{13}\right]}\right) \\
& +\left(\hat{I}^{+}\right)^{*} e^{i\left(2 \varphi_{E}-\Psi_{S E}+\Psi_{E 2}-\Phi_{S 2}\right)}\left(1+e^{i\left[2\left(\varphi_{W}-\varphi_{N}\right)-\Phi_{24}\right]}\right) .
\end{aligned}
$$

This shows that it is always possible to cancel one of the spin-orbit coupling integrals by choosing the snake angles so that the spin perturbation produced in one of the arcs is canceled by the arc on the opposite side of the ring. Since $\left|\hat{I}^{+}\right|$and $\left|\hat{I}^{-}\right|$are different, neighboring arcs can in general not compensate each other when four Siberian snakes are used.

It is however possible to use the eight-snake scheme found for symmetric lattices. The two special eight Siberian snake schemes which lead to an energy independent snake match in a ring with superperiodicity 4 will not spin match HERA- $p$ completely, but the spin perturbation from the arcs, which are the dominant perturbation, will be compensated exactly. This possibility of having a set of Siberian snake angles which do not have to be changed with energy and which lead to a tightly bundled invariant spin field is on the one hand very attractive; on the other hand it requires eight Siberian snakes of which four would have to be installed at the centers of the HERA- $p$ arcs, where technical requirements of moving cryogenic feedthroughs and superconducting magnets would be very costly. If possible, a foursnake scheme should therefore be found.

Whereas it was shown below (18) that a four-snake scheme cannot cancel both spin-orbit-coupling integrals in a ring with superperiodicity, a corresponding cancellation of the spin perturbation due to the arcs in HERA- $p$ can nevertheless be achieved since the orbital phase advances between the arcs can be manipulated individually, while these four phase advances are equal for a lattice with superperiodicity 4 .

To cancel both spin-orbit integrals in (42), four phase factors have to be -1 . This requires

$$
\begin{aligned}
& 2\left(\varphi_{E}-\varphi_{N}\right)+\Phi_{13} \stackrel{\circ}{=} \pi, \\
& 2\left(\varphi_{E}-\varphi_{N}\right)-\Phi_{13} \stackrel{\circ}{=} \pi, \\
& 2\left(\varphi_{W}-\varphi_{N}\right)+\Phi_{24} \stackrel{\circ}{=} \pi,
\end{aligned}
$$

$$
2\left(\varphi_{W}-\varphi_{N}\right)-\Phi_{24} \stackrel{\circ}{=} \pi
$$

Subtraction of the first two equations leads to the requirement that the betatron phase advance from $\theta_{1}$ halfway around the ring to $\theta_{3}$ is an odd or even multiple of $\pi$. The same is true for the phase advance from $\theta_{2}$ to $\theta_{4}$. Correspondingly, the spin phase advance over these regions has to be an odd multiple of $\pi$ when the orbit phase advance is an even multiple and vice versa. With a rather benign change of the vertical optics in HERA- $p$ which does not change the vertical tune, the contribution of the regular arcs to both spin-orbit-coupling integrals can be canceled, even in a four-snake scheme.

The snake scheme $\left(0 \frac{\pi}{2} \frac{\pi}{2} \frac{\pi}{2}\right) o$ has $\Psi_{13}=0$ and $\Psi_{24}=$ 0 . For this snake scheme, the betatron phase advances from $\theta_{1}$ to $\theta_{3}$ and from $\theta_{2}$ to $\theta_{4}$ were adjusted to be odd multiples of $\pi$. This change of the optics is indicated by the index $o$ in the notation of the snake scheme. The maximum time average polarization $P_{\lim }$ is plotted (blue) in Fig. 9 for the complete range of HERA- $p$ momenta (top) and for the critical momentum regions above $800 \mathrm{GeV} / c$ (bottom). As a comparison, $P_{\lim }$ for a standard snake scheme $\left(\frac{\pi}{4} 0 \frac{\pi}{4} 0\right)$ (red) is also shown.

The complete snake match of the arcs in HERA- $p$ indeed eliminates all strong reductions of $P_{\lim }$ over the complete momentum range. Nonlinear effects will be analyzed later, but as far as the linear effects are concerned, this snake-matched lattice of HERA- $p$ would be a rather promising choice for the acceleration of polarized proton beams.

\section{B. Schemes with eight snakes}

Although, for the reasons explained, eight snakes are not very practical for HERA- $p$, significant improvements would be possible if eight snakes were used, as will now be shown. The snake matching schemes of Fig. 7 are suitable for rings with superperiodicity 4 . They are therefore not directly applicable to HERA- $p$. However, the cancellation schemes of Fig. 6 can still provide a guide to snake matching HERA- $p$ with eight snakes.

The eight Siberian snakes are placed in the straight sections and into the centers of the arcs, so that the horizontal angle between adjacent Siberian snakes is $45^{\circ}$ and therefore $\Psi_{j}=G \gamma \pi / 4$ for all $j \in\{0 \cdots 7\}$. This ensures that the snake match is independent of energy since then the $n$th octant's contribution $I_{n-1}^{ \pm}$to the spin-orbit coupling integral $I^{ \pm}$can be compensated by the second neighbor's contributions $I_{n+1}^{ \pm}$. In particular, the $\Psi_{j}$ cancel in the phase factor in

$$
I_{n-1}^{ \pm}+I_{n+1}^{ \pm} e^{i\left[(-1)^{n}\left(\Psi_{n-1}-\alpha_{n}-\Psi_{n}+\alpha_{n+1}\right) \pm\left(\Phi_{n-1}+\Phi_{n}\right)\right]} .
$$

Now a snake scheme for HERA- $p$ is sought that is guided by the cancellation scheme of Fig. 6 (left). When the first octant's contribution $I_{0}^{ \pm}$to a spin-orbit coupling integral $I^{ \pm}$is to be compensated by that of its second 

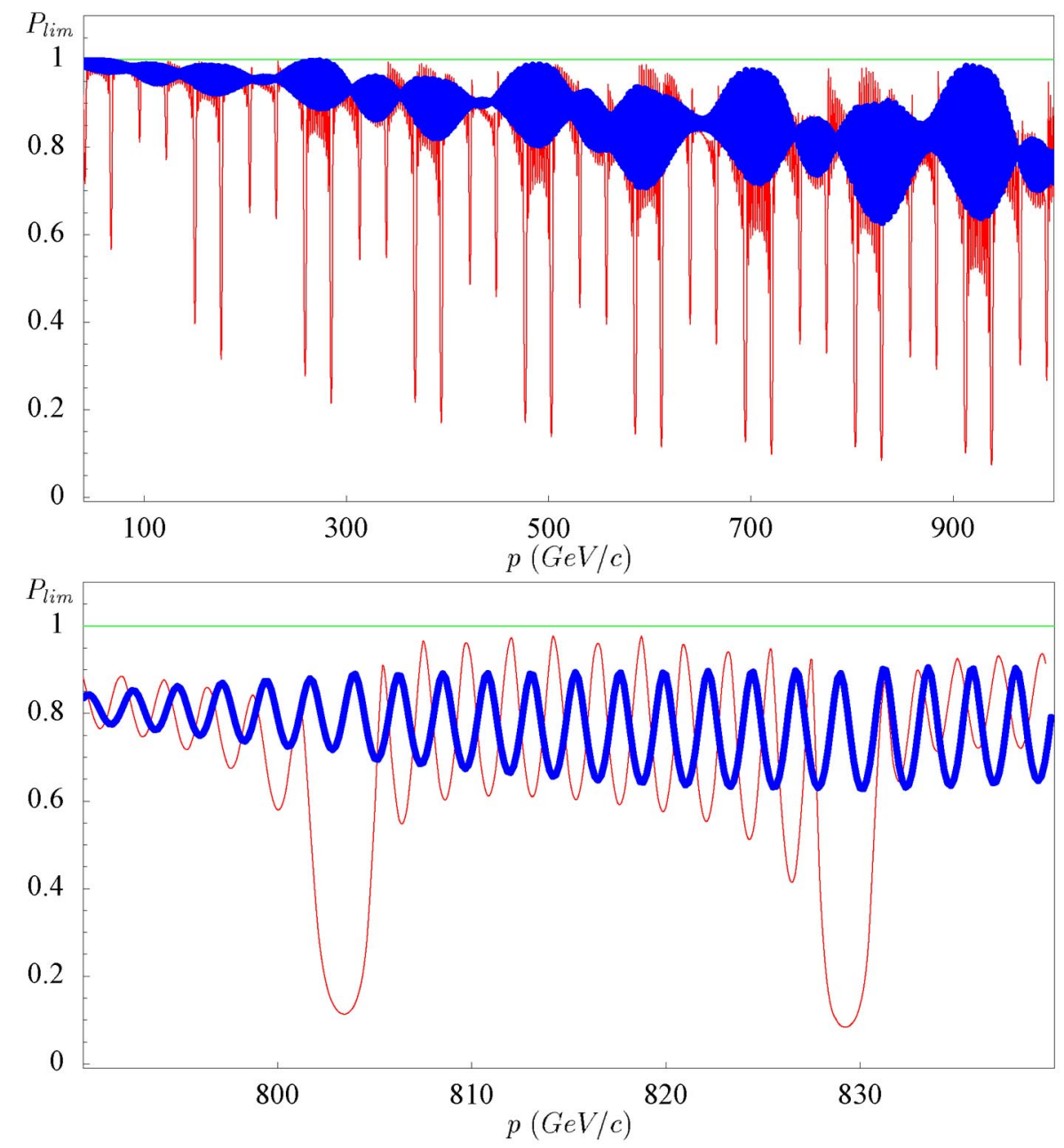

FIG. 9. (Color) Improvement of $P_{\lim }$ by matching four snake angles and the orbital phases. The snake arrangement is $\left(0 \frac{\pi}{2} \frac{\pi}{2} \frac{\pi}{2}\right) o$ (blue). As a comparison $P_{\text {lim }}$ from linearized spin-orbit motion is shown for the same HERA- $p$ optics with a $\left(\frac{\pi}{4} 0 \frac{\pi}{4} 0\right) o$ snake scheme (red).

neighbor $I_{2}^{ \pm}$, the phase factor in

$$
I_{0}^{ \pm}+e^{i\left[-\Psi_{0}+\alpha_{1}+\Psi_{1}-\alpha_{2} \pm\left(\Phi_{0}+\Phi_{1}\right)\right]} I_{2}^{ \pm}
$$

should be -1 . The same should be true for the phase factor in

$$
I_{4}^{ \pm}+e^{i\left[-\Psi_{4}+\alpha_{5}+\Psi_{5}-\alpha_{6} \pm\left(\Phi_{4}+\Phi_{5}\right)\right]} I_{6}^{ \pm} .
$$

These conditions are satisfied for the superscript + as well as - when all the snake angles $\alpha_{j}$ are zero for $j \in$ $\{1,2,5,6\}$ and the betatron phase advances are chosen appropriately. Similar conditions arise for the phase factors involved in matching $I_{1}$ against $I_{3}$ and in matching $I_{5}$ against $I_{7}$. They are also -1 when the betatron phases are chosen appropriately and when $\alpha_{3}$ and $\alpha_{7}$ are zero. Since $\alpha_{0}$ and $\alpha_{4}$ do not appear in these matching conditions, they can be chosen freely. But for a design orbit spin tune of 0.5 , one has to choose $\alpha_{0}+\alpha_{4}=\pi$. Here $\alpha_{4}=0$ has been chosen and this snake scheme is then characterized as $\left(\frac{\pi}{2} 0000000\right) o$.
In the case of HERA- $p$ spin-orbit integrals of complete octants cannot cancel, but the contribution of the regular arc sections can cancel. Therefore the betatron phase advance is not chosen to be $\Phi_{0}+\Phi_{1} \stackrel{\circ}{=} \pi$ between Siberian snakes, but rather the betatron phase advances between the beginning of the regular arcs of the first and the second quadrant are chosen in that way, i.e., $\Phi_{12} \stackrel{\circ}{=} \pi$ with the notation of Fig. 8. In this configuration the regular arc of the first octant cancels that of the third, i.e., the regular arc's contribution to $I_{2}$ cancels that to $I_{0}$. But also the regular arc of the second octant cancels that of the fourth since the betatron phase advance between the centers of the first and the second arc is then also $\pi \bmod 2 \pi$, i.e., the regular arc part of $I_{3}$ cancels that of $I_{1}$. Similarly, the octants of the third quadrant cancel those of the fourth quadrant. In the snake scheme $\left(\frac{\pi}{2} 0000000\right) o$ the phase advance over the east straight section was changed by $2 \pi \times 0.1028$ to have $\Phi_{12}=2 \pi \times 8.5$. The phase advance over the west was changed by $2 \pi \times 0.0208$ 
to have $\Phi_{34}=2 \pi \times 7.5$. In order to have the same betatron phase advance in the north and the south straight sections and to keep the total tune constant, the linear optics of the north and of the south straight sections were modified.

This cancellation scheme does not agree completely with that of Fig. 6 (left) since in $\left(\frac{\pi}{2} 0000000\right) o$ the superscripts + and - have been dealt with simultaneously so that $I_{0}^{\mp}$ no longer has to compensate $I_{4}^{\mp}$. Since $\Phi_{12} \stackrel{\circ}{=} \pi$ and $\Phi_{34} \stackrel{\circ}{=} \pi$, it is appropriate to take $q=\pi / 2$ for the phase advance of one octant of the scheme in Fig. 7 (left), for which one then finds a close similarity to the snake scheme $\left(\frac{\pi}{2} 0000000\right) o$. The resemblance would be even closer if the phase advance over the north straight section had been changed so that $\Phi_{23} \stackrel{\circ}{=} 0$ and if $\alpha_{4}=\pi, \alpha_{0}=0$ had been chosen. This would require an additional change of $\Phi_{41}$ to adjust the orbital tune.

A snake scheme for HERA- $p$ which resembles Fig. 6 (right) can also be found. In this cancellation scheme the phase factors in the following sums have to be -1 :

$$
\begin{gathered}
I_{0}^{+}+e^{i\left[-\Psi_{0}+\alpha_{1}+\Psi_{1}-\alpha_{2}+\left(\Phi_{0}+\Phi_{1}\right)\right]} I_{2}^{ \pm}, \\
I_{4}^{+}+e^{i\left[-\Psi_{4}+\alpha_{5}+\Psi_{5}-\alpha_{6}+\left(\Phi_{4}+\Phi_{5}\right)\right]} I_{6}^{ \pm}, \\
I_{0}^{-}+e^{i\left(-\Psi_{0}+\alpha_{1}+\Psi_{1}-\alpha_{2}-\Psi_{2}+\alpha_{3}+\Psi_{3}-\alpha_{4}\right)} e^{-i\left(\Phi_{0}+\Phi_{1}+\Phi_{2}+\Phi_{3}\right)} I_{4}^{ \pm},
\end{gathered}
$$

$I_{2}^{-}+e^{i\left(-\Psi_{2}+\alpha_{3}+\Psi_{3}-\alpha_{4}-\Psi_{4}+\alpha_{5}+\Psi_{5}-\alpha_{6}\right)} e^{-i\left(\Phi_{2}+\Phi_{3}+\Phi_{4}+\Phi_{5}\right)} I_{6}^{ \pm}$,

$$
I_{1}^{-}+e^{i\left[\Psi_{1}-\alpha_{2}-\Psi_{2}+\alpha_{3}-\left(\Phi_{1}+\Phi_{2}\right)\right]} I_{3}^{ \pm}
$$

$$
\begin{gathered}
I_{5}^{-}+e^{i\left[\Psi_{5}-\alpha_{6}-\Psi_{6}+\alpha_{7}-\left(\Phi_{5}+\Phi_{6}\right)\right]} I_{7}^{ \pm}, \\
I_{1}^{+}+e^{i\left(\Psi_{1}-\alpha_{2}-\Psi_{2}+\alpha_{3}+\Psi_{3}-\alpha_{4}-\Psi_{4}+\alpha_{5}\right)} e^{i\left(\Phi_{1}+\Phi_{2}+\Phi_{3}+\Phi_{4}\right)} I_{5}^{ \pm}, \\
I_{3}^{+}+e^{i\left(\Psi_{3}-\alpha_{4}-\Psi_{4}+\alpha_{5}+\Psi_{5}-\alpha_{6}-\Psi_{6}+\alpha_{7}\right)} e^{i\left(\Phi_{3}+\Phi_{4}+\Phi_{5}+\Phi_{6}\right)} I_{7}^{ \pm} .
\end{gathered}
$$

Again, complete octants cannot cancel, but to cancel the contributions of regular arc sections the beginning of a regular arc is chosen as the starting point for the compensation. Now the $\Phi_{n}$ for all even $n$ describe the betatron phase advance from the beginning to the center of the regular arc, and they are therefore equal. For the choice $\alpha_{4}=0$ all phase factors are -1 if and only if $\Phi_{5}=\Phi_{1}$ and

$$
\begin{array}{rrr}
\alpha_{1} \stackrel{\circ}{=} \Phi_{23}, & \alpha_{2} \stackrel{\circ}{=} \Phi_{13}, & \alpha_{3} \stackrel{\circ}{=} \Phi_{14}, \\
\alpha_{5}=-\alpha_{3}, & \alpha_{6}=-\alpha_{2}, & \alpha_{7}=-\alpha_{1} .
\end{array}
$$

This snake scheme is referred to as $\left(\frac{\pi}{2} a b c 0-c-b-a\right) o$. The condition $\Phi_{5}=\Phi_{1}$ was satisfied by changing the betatron phase advance over the east and west straight section without changing the vertical tune.

Linearized spin-orbit motion leads to a very favorable $P_{\text {lim }}$ for both eight-snake schemes as shown in Fig. 10, where it is compared to the $P_{\text {lim }}$ of the snake and phase advance matched four-snake scheme $\left(0 \frac{\pi}{2} \frac{\pi}{2} \frac{\pi}{2}\right) o$.

\section{HIGHER-ORDER RESONANCES AND SNAKE SCHEMES}

At the critical energies, where the maximum time average polarization is low during the acceleration pro-

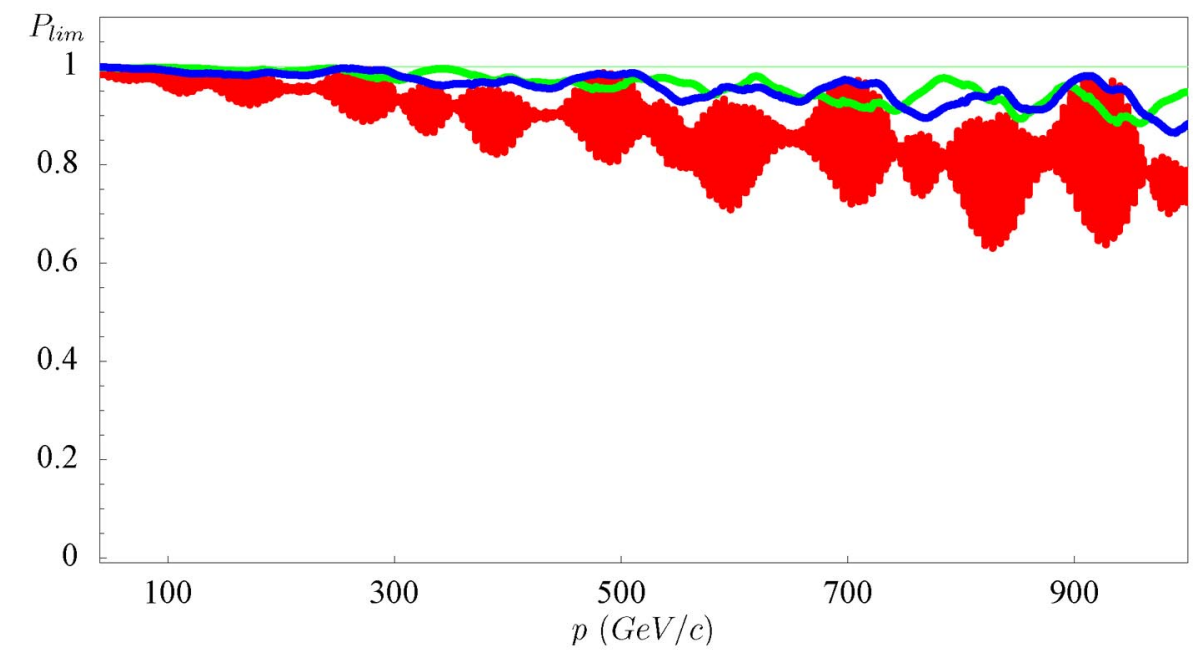

FIG. 10. (Color) Improvement of $P_{\lim }$ by matching eight snake angles and the orbital phases. The snake arrangement is $\left(\frac{\pi}{2} 0000000\right) o$ (blue) and $\left(\frac{\pi}{2} a b c 0-c-b-a\right) o$ (green). As a comparison $P_{\text {lim }}$ from linearized spin-orbit motion is shown for the snake and phase advance matched four-snake scheme $\left(0 \frac{\pi}{2} \frac{\pi}{2} \frac{\pi}{2}\right) o$ (red). 
cess, linearized spin-orbit motion does not describe spin dynamics well. The spin motion is influenced by several overlapping resonances in these regions and the single resonance approximations $[16,34]$ can also not be applied. Thus the simulation results obtained with these computationally quick techniques should always be checked with more time-consuming nonperturbative methods if possible. This is also true for the snake-matched lattices of HERA- $p$ with large $P_{\text {lim }}$, even though they avoid large variations of the invariant spin field $\vec{n}(\vec{z})$ over the phase space of the beam in linearized spin-orbit motion. When first-order effects are canceled, the higher-order effects become dominant and the quality of the snake-matched lattice of HERA- $p$ can only be evaluated with higherorder theories.

Until 1996, when stroboscopic averaging [13] was introduced, there was no nonperturbative method of computing the $\vec{n}$ axis at high energy in proton storage rings, where perturbative methods are usually not sufficient [14]. In addition, the method of antidamping was derived [35], which also computes $\vec{n}(\vec{z})$ nonperturbatively and which can be faster when the $\vec{n}$ axis is required for a range of phase space amplitudes. Both methods of computing the invariant spin field are implemented in the spin-orbit dynamics code SPRINT, by which also the amplitude-dependent spin tune $\nu(\vec{J})$ can be computed once $\vec{n}(\vec{z})$ is known. Since stroboscopic averaging and antidamping are based on multiturn tracking data, they are applicable to all kinds of circular accelerators and they are especially efficient for small rings and for simple model accelerators. Nonlinear motion for orbit and spin coordinates can easily be included $[36,37]$.
Subsequently, another nonperturbative algorithm for computing $\vec{n}(\vec{z})$ and $\nu(\vec{J})$ has been derived [38]. It is called SODOM-2 since it was inspired by the earlier algorithm SODOM [11], which for convergence required the angle between $\vec{n}$ and $\vec{n}_{0}$ to be small. With some routines provided by Yokoya, SODOM-2 was incorporated into the program SPRINT $[13,39,40]$ and leads to results which agree very well with those of stroboscopic averaging. For motion in 1 degree of freedom, SODOM- 2 is often faster than stroboscopic averaging, especially for large rings like HERA- $p$ where particle tracking is relatively time consuming. But for orbit motion in more than 1 degree of freedom or in the vicinity of spin-orbit resonances, SODOM-2 becomes exceedingly slow and then stroboscopic averaging and antidamping are needed.

To check whether the improvements of spin motion obtained in the framework of linearized spin-orbit motion survive when higher-order effects are considered, $P_{\text {lim }}$ and $\nu$ have been calculated. The result for one of the standard Siberian snake schemes which used to be considered advantageous by popular opinion is shown for the south interaction point of HERA- $p$ in Fig. 11. It has four Siberian snakes in the $\left(\frac{\pi}{4} 0 \frac{\pi}{4} 0\right)$ scheme. Some of the features of $P_{\lim }$ were already revealed by linearized spinorbit motion in Fig. 1. Now many higher-order resonances are revealed, causing strong reduction of $P_{\lim }$ and there are corresponding strong variations of the amplitudedependent spin tune $\nu$ [41]. Strong resonances occur especially in the critical energy region where linearized spin-orbit motion in Sec. II already indicated a very small $P_{\text {lim }}$ due to a coherent spin perturbation in all regular FODO cells. Many higher-order resonances overlap in
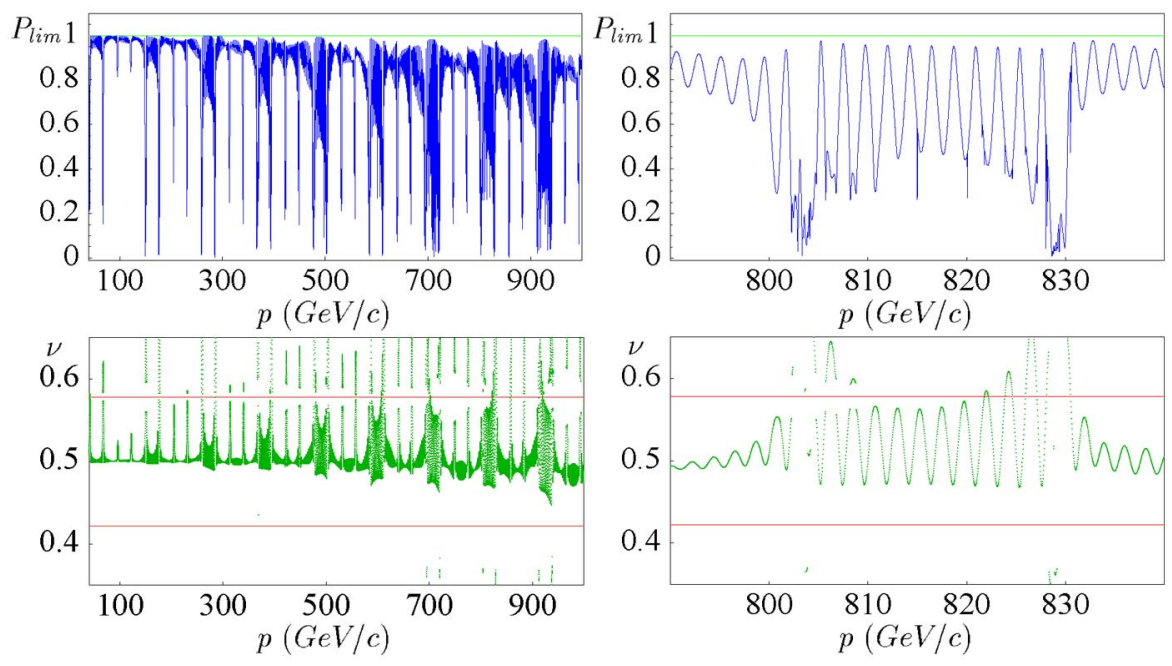

FIG. 11. (Color) $P_{\lim }$ and $\nu$ for particles with a vertical amplitude corresponding to the $2.5 \sigma$ emittance in the HERA- $p$ lattice of the year 2002 with the $\left(\frac{\pi}{4} 0 \frac{\pi}{4} 0\right)$ scheme. Top: the maximum time average polarization $P_{\text {lim }}$ for the complete acceleration range (left) and for the critical energy range above $800 \mathrm{GeV} / c$ which has to be crossed when accelerating to the proposed storage energy of $870 \mathrm{GeV} / c$. Bottom: the corresponding amplitude-dependent spin tune $\nu\left(J_{y}\right)$. The second-order resonances $\nu=2 Q_{y}$ and $\nu=$ $1-2 Q_{y}$ are indicated (red). 
these critical energy regions of Fig. 11 (top) where large spin tune jumps can be observed in Fig. 11 (bottom). The strongest spin tune jumps occur in the critical energy regions, mostly at the second-order resonance $\nu=2 Q_{y}$ which is indicated by the top line [42].

Figure 12 also shows $P_{\lim }$ and $\nu$ for higher-order spin dynamics in the snake-matched and phase-advancematched HERA- $p$ ring with four Siberian snakes. While the overall behavior of $P_{\text {lim }}$ over the complete acceleration range of HERA- $p$ looks similar to the result obtained with linearized spin-orbit motion, which was displayed in Fig. 9, higher-order effects become very strong at high energies, especially in the vicinity of the critical energies where perturbations of spin motion in each FODO cell accumulate. The spin tune spread at momenta below $400 \mathrm{GeV} / c$ is small and higher-order effects seem to be benign even at these critical energies. A comparison of Figs. 11 and 12 shows that the special scheme obtained by matching orbital phases and snake angles would be a very good choice for HERA- $p$ up to 300 or $400 \mathrm{GeV} / c$. For linearized spin-orbit motion, this snake-matched scheme does not produce a strong reduction of $P_{\text {lim }}$ at any critical energy, but the higher-order effects become very pronounced at some top energies of HERA- $p$. Nevertheless, the advantage over other snake schemes becomes clear in Fig. 12 (bottom) where the amplitude-dependent spin
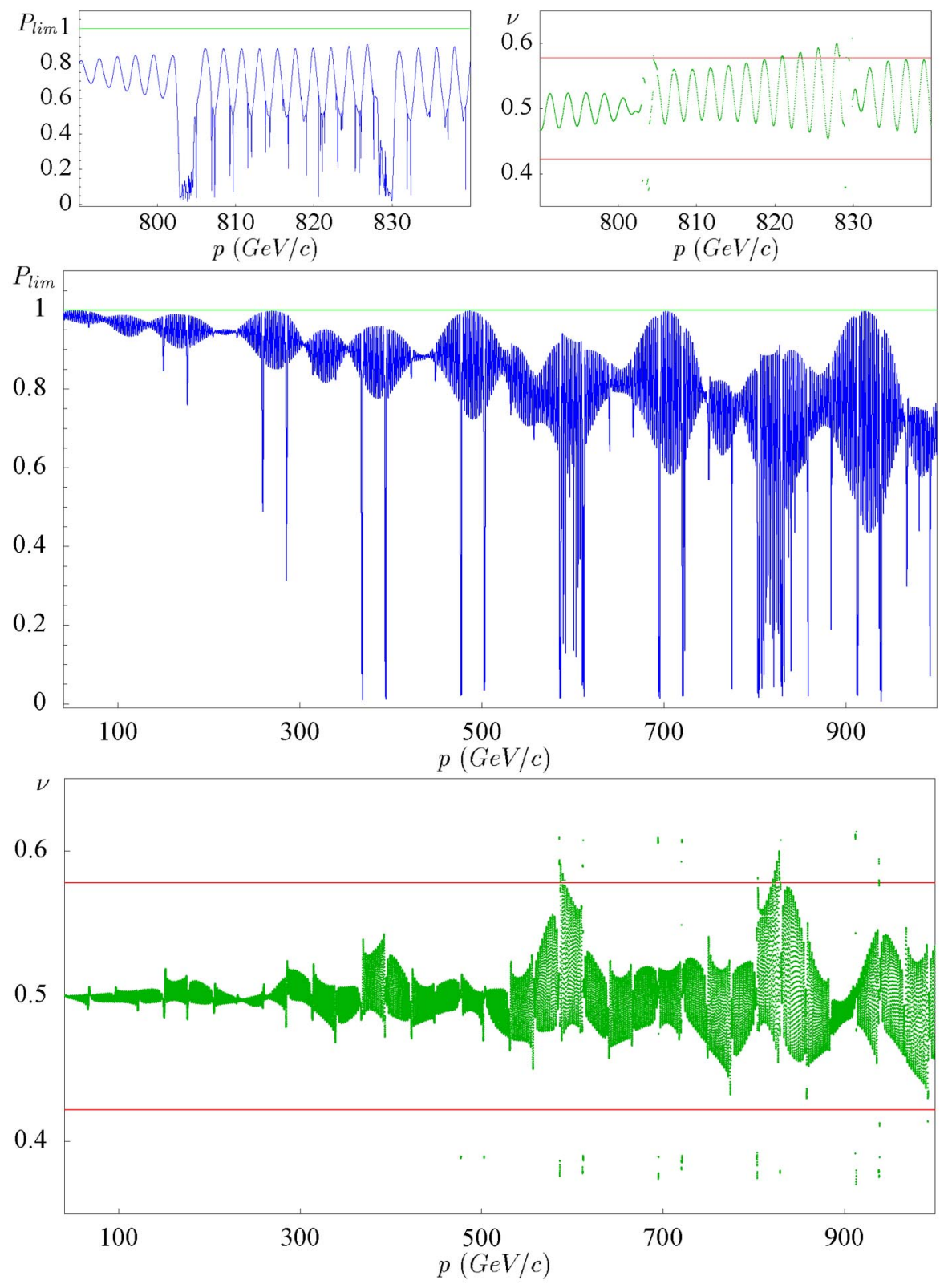

FIG. 12. (Color) $P_{\lim }$ and $\nu$ for a $2.5 \sigma$ vertical amplitude after the betatron phase advance between opposite regular arc structures was adjusted to be an odd multiple of $\pi$ in the $\left(0 \frac{\pi}{2} \frac{\pi}{2} \frac{\pi}{2}\right) o$ scheme. The resonances $\nu=2 Q_{y}$ and $\nu=1-2 Q_{y}$ are indicated (red). 
tune $\nu$ is shown. It comes close to a second-order resonance at fewer places and does not exhibit spin tune jumps which are as strong as those in previous figures of $\nu$.

In this snake-matched scheme, the influence of higherorder effects can be seen very clearly, because the firstorder effects have been matched to be very small. The analysis of $\nu$ shows that completely snake matching the spin perturbations in the arcs of HERA- $p$ with four Siberian snakes is advantageous, even though dips of $P_{\lim }$ due to higher-order resonances can be observed at high energies. Even around $300 \mathrm{GeV} / c$ there are resonant dips of $P_{\text {lim }}$ in Fig. 12 (middle) but they are less pronounced than those in Fig. 11 so that the snake-matched scheme should be very advantageous in the complete energy range of HERA- $p$.

\section{POLARIZATION REDUCTION DURING ACCELERATION}

It should be noted that the destructive spin tune jumps at second-order resonances disappear completely when HERA- $p$ is simulated without its nonflat regions. This is due to the fact that a large class of resonances is not excited at all in midplane symmetric rings [5]. To reduce these perturbations, the east region of HERA- $p$ will now be simulated as flat since the HERMES experiment located in this region does not require that the proton beam is on the level of the electron beam.

When a particle is accelerated across the critical momentum region from 800 to $806 \mathrm{GeV} / c$ with a typical acceleration rate of $50 \mathrm{keV}$ per turn, the adiabatic invariance of $J_{S}=\vec{n} \cdot \vec{S}$ can be violated and the level of violation will depend on the orbital amplitude and the snake scheme. This violation is illustrated in the graphs in Fig. 13 which, for three different snake schemes, show the average spin action $\bar{J}_{S}$ at $806 \mathrm{GeV} / c$ which had initially $J_{S}=1$ at $800 \mathrm{GeV} / c$ before acceleration.

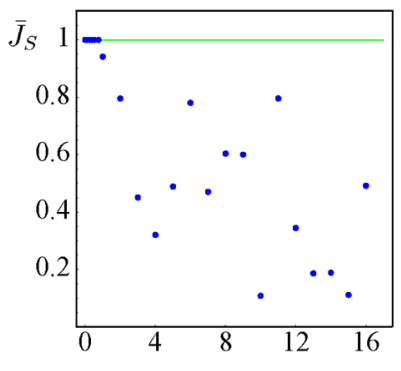

Amplitude ( $\pi \mathrm{mm}$ mrad)

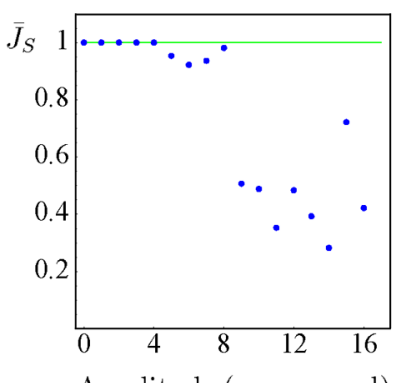

Amplitude ( $\pi \mathrm{mm}$ mrad $)$
FIG. 13. (Color) Average spin action $\bar{J}_{S}$ at $806 \mathrm{GeV}$ for particles starting with $J_{S}=1$ at $800 \mathrm{GeV} / c$ for three different snake schemes. Left: $\left(\frac{\pi}{4} 0 \frac{\pi}{4} 0\right)$; middle: $\left(\frac{3 \pi}{4} \frac{3 \pi}{8} \frac{3 \pi}{8} \frac{\pi}{4}\right) o$; right: $\left(0 \frac{\pi}{2} \frac{\pi}{2} \frac{\pi}{2}\right) o$ scheme. Particles with an amplitude above 1 (left), 4 (middle), and 8 (right) lead to a reduction of polarization when the beam is accelerated through this critical energy region.
The change of $J_{S}$ in the critical energy region depends on the initial phase space angle so that if $J_{S}$ had been computed only for one particle, it could by chance have had an angle variable for which $J_{S}$ does not change although it would have changed for other points with the same vertical phase space amplitude. To avoid such a chance effect which gives the impression that $J_{S}$ is invariant, three particles were accelerated and the average $\bar{J}_{S}$ is displayed in Fig. 13.

At small phase space amplitudes, $J_{S}$ is nearly invariant and therefore $\bar{J}_{S}=1$. For each of the three snake schemes, there is a phase space amplitude $J_{y \max }$ above which $\bar{J}_{S}<1$ and the regions of the beam with an amplitude above $J_{y \max }$ lead to a reduction of the beam's polarization during the acceleration process.

For the standard snake scheme $\left(\frac{\pi}{4} 0 \frac{\pi}{4} 0\right)$, only the part of the beam with less than $1 \pi \mathrm{mm}$ mrad vertical amplitude can remain polarized. For the scheme $\left(\frac{3 \pi}{4} \frac{3 \pi}{8} \frac{3 \pi}{8} \frac{\pi}{4}\right) O$ that was obtained by numerical optimization, phase space amplitudes up to $4 \pi \mathrm{mm}$ mrad are allowed. Finally the snake-matched scheme $\left(\frac{\pi}{4} 0 \frac{\pi}{4} \frac{\pi}{4}\right) o$ gives the most stable spin motion and Fig. 13 shows that vertical amplitudes of up to $8 \pi \mathrm{mm}$ mrad are allowed.

This shows that the snake-matched scheme is superior to the other four-snake schemes studied here. It stabilizes spin motion for 10 times larger phase space amplitudes than some other snake schemes. Nevertheless, $8 \pi \mathrm{mm} \mathrm{mrad}$ is not enough to allow high polarization at top energies for today's emittances in HERA- $p$.

Matching eight Siberian snakes in HERA- $p$ lead to two snake schemes with very high $P_{\text {lim }}$ and very small spin tune spread. But to demonstrate that it is possible to further stabilize spin motion in HERA- $p$ by such schemes, Fig. 14 shows the vertical phase space amplitudes for which $J_{S}$ remains invariant. The more effective

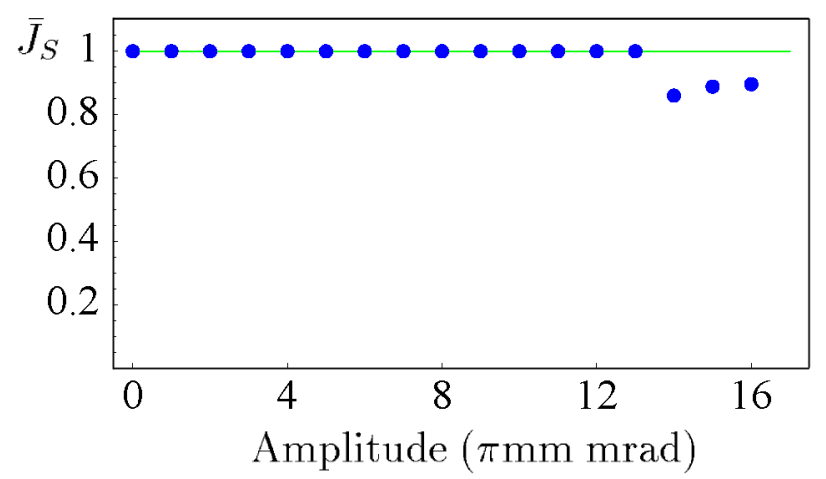

FIG. 14. (Color) The average spin action $\bar{J}_{S}$ at $806 \mathrm{GeV}$ for particles which started with $J_{S}=1$ at $800 \mathrm{GeV} / c$ for the two different snake-matched eight-snake schemes $\left(\frac{\pi}{2} 0000000\right) o$ (left) and $\left(\frac{\pi}{2} a b c 1-c-b-a\right) o$ (right). Particles with an amplitude above 13 (left) and 15 (right) lead to a reduction of polarization when the beam is accelerated through this critical energy region. 


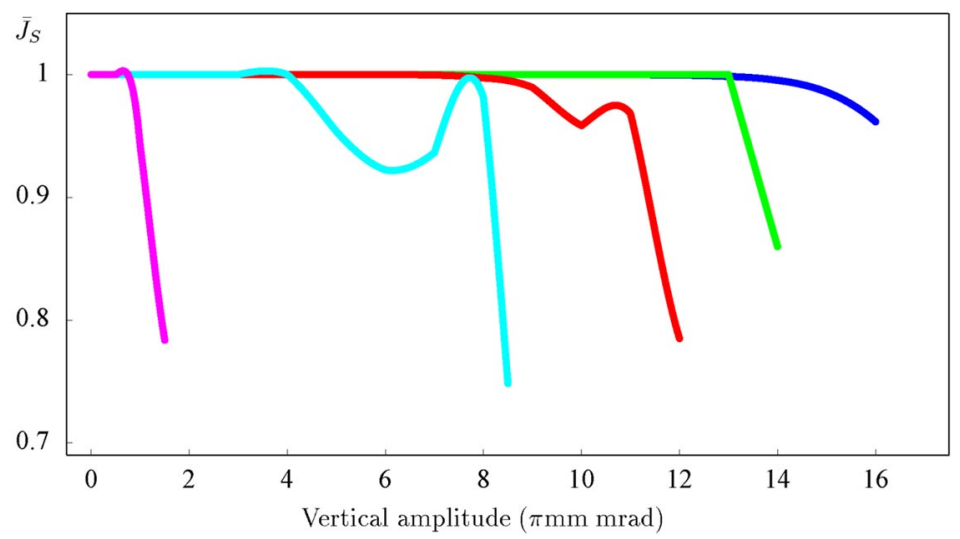

FIG. 15. (Color) The average spin action $\bar{J}_{S}$ at $806 \mathrm{GeV}$ for particles which started initially with $J_{S}=1$ at $800 \mathrm{GeV} / c$. Violet: the standard scheme which stabilizes spin motion for particles within $1 \pi \mathrm{mm} \mathrm{mrad}$; cyan: the four-snake scheme from numerical optimization stabilizes within $4 \pi \mathrm{mm}$ mrad; red: the snake-matched four-snake scheme stabilizes within $8 \pi \mathrm{mm}$ mrad; green: the snake-matched eight-snake scheme which stabilizes within $13 \pi \mathrm{mm}$ mrad; blue: the snake-matched eight-snake scheme which stabilizes within $14 \pi \mathrm{mm}$ mrad of vertical phase space amplitude.
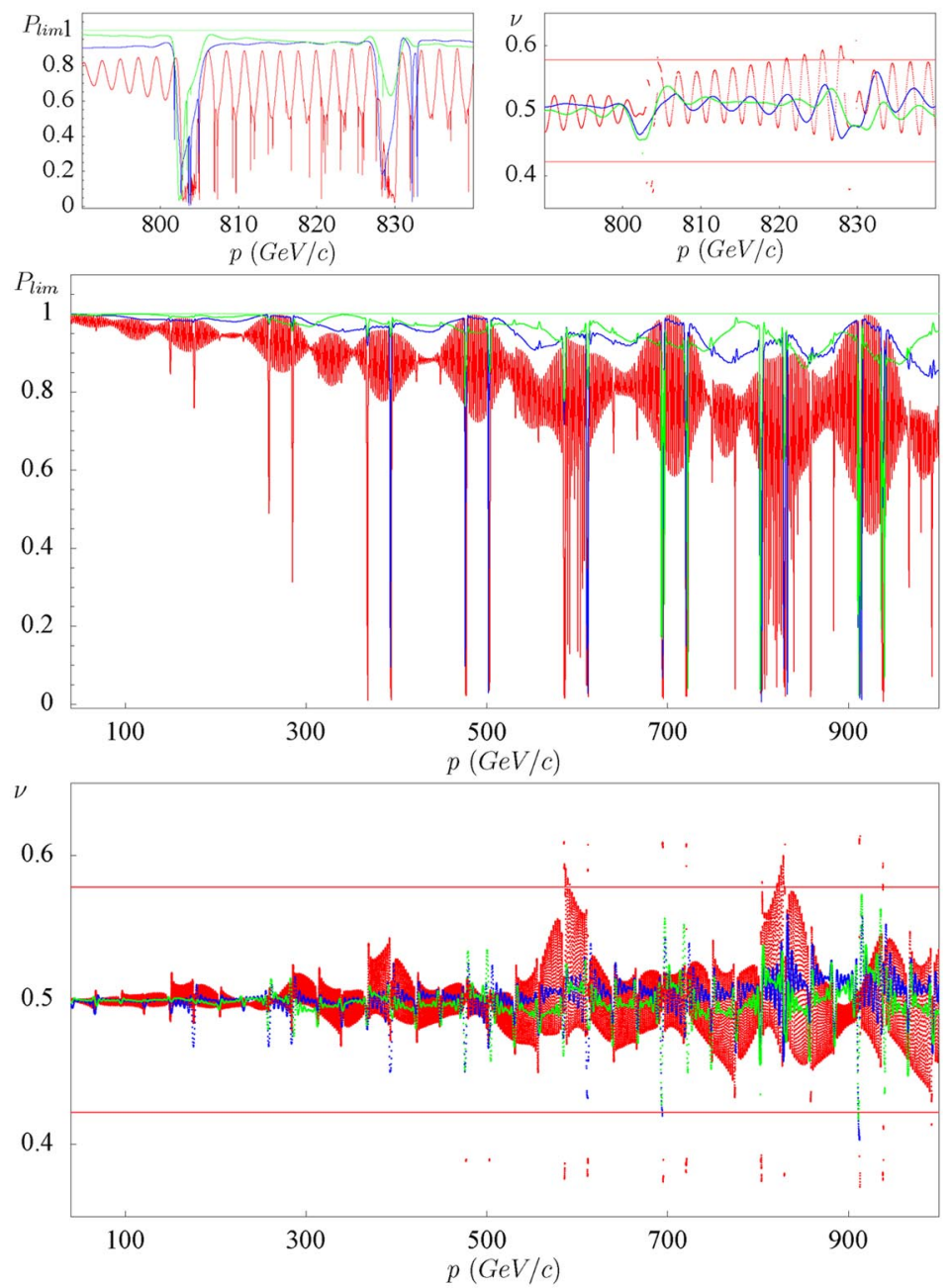

FIG. 16. (Color) Improvement of the higher-order $P_{\text {lim }}$ and $\nu\left(J_{y}\right)$ by matching eight snake angles and the orbital phases. The snake arrangement is $\left(\frac{\pi}{2} 0000000\right) o$ (blue) and $\left(\frac{\pi}{2} a b c 0-c-b-a\right) o$ (green). As a comparison $P_{\text {lim }}$ from soDOM-2 is shown for the snake and phase advance matched four-snake scheme $\left(0 \frac{\pi}{2} \frac{\pi}{2} \frac{\pi}{2}\right) o$ (red background curve). The resonances $\nu=2 Q_{y}$ and $\nu=1-2 Q_{y}$ are indicated (also red). 
of the two-snake scheme stabilizes spin motion up to a vertical amplitude of $14 \pi \mathrm{mm}$ mrad.

These results for the various snake schemes are collected in Fig. 15, where it becomes clear that snake matching with four and especially with eight snakes leads to a significant improvement. In HERA- $p$ it does not suffice to avoid a reduction of $J_{S}$ for particles with less than $14 \pi \mathrm{mm}$ mrad amplitude. It would therefore be very helpful to use electron cooling in PETRA [43-45] so as to reduce the emittance in HERA- $p$ and to allow for an acceleration without loss of polarization for most particles in the beam.

The excellent performance of the two schemes with eight Siberian snakes is due to much smaller oscillation of the amplitude-dependent spin tune during the acceleration process, and the destructive second-order spin-orbit resonances indicated in Fig. 16 (bottom and top-right) are hardly encountered when these snake schemes are chosen. Correspondingly, $P_{\lim }$ only drops to small values at very few energies in Fig. 16 (center and top-left).

Contemplating all these results, it can be concluded that it is not possible to give a simple formula for the number of snakes which are required for a given accelerator since different snake schemes with the same number of snakes lead to very different stability of spin motion. It has even been shown that eight snakes are not necessarily better than four snakes for the nonflat HERA- $p$ ring [46]. In the end, detailed evaluation is needed.

\section{ACKNOWLEDGMENTS}

Desmond Barber's careful reading and improving of the manuscript are thankfully acknowledged.

[1] K. Steffen, DESY Report No. DESY-88-068 (1988).

[2] E. D. Courant, S. Y. Lee, and S. Tepikian, in Polarized Beams at SSC \& Polarized Antiprotons, edited by A. D. Krisch, A.M.T. Lin, and O. Chamberlain, AIP Conf. Proc. No. 145 (AIP, New York, 1986), p. 174.

[3] D. P. Barber, K. Heinemann, G. H. Hoffstaetter, and M. Vogt, in Proceedings of the ICFA Beam Dynamics Workshop on Quantum Aspects of Beam Physics, Monterey, California, 1998 (World Scientific, Singapore, 1998).

[4] G. H. Hoffstaetter, Habilitation, Darmstadt University of Technology, 2000.

[5] M. Vogt, Ph.D. dissertation, Universität Hamburg [DESY Report No. DESY-THESIS-2000-054, 2000].

[6] M. Vogt, D. P. Barber, and G. H. Hoffstaetter, DESY Report No. DESY-HERA-98-04, 1998.

[7] G. H. Hoffstaetter, H.S. Dumas, and J.A. Ellison, in Proceedings of the European Particle Accelerator Conference (EPAC02), Paris, 2002 (EPS-IGA and CERN, Geneva, 2002); DESY Report No. DESY-M-0201, 2002.
[8] A.W. Chao, Nucl. Instrum. Methods 180, 29 (1981).

[9] Yu. Eidelman and V. Yakimenko, Part. Accel. 45, 17 (1994).

[10] S. R. Mane, Phys. Rev. A 36, 120 (1987).

[11] K. Yokoya, KEK Report No. KEK-92-6, 1992.

[12] V. Balandin and N. Golubeva, in Proceedings of the Conference on High Energy Accelerators, Hamburg, 1992 (unpublished), Vol. 2, pp. 998-1000.

[13] K. Heinemann and G. H. Hoffstaetter, Phys. Rev. E 54, 4240 (1996).

[14] G. H. Hoffstaetter, M. Vogt, and D. P. Barber, Phys. Rev. ST Accel. Beams 2, 114001 (1999).

[15] D. P. Barber, G. H. Hoffstaetter, and M. Vogt, in Proceedings of the European Particle Accelerator Conference (EPAC02), Paris, 2002 (Ref. [7]); DESY Report No. DESY-M-02-01, 2002.

[16] M. Froissart and R. Stora, Nucl. Instrum. Methods 7, 297 (1960).

[17] V. Balandin, N. Golubeva, and D. P. Barber, DESY Report No. DESY-M-96-04, 1996.

[18] Ya. S. Derbenev and A. M. Kondratenko, Dokl. Akad. Nauk SSSR 223, 830 (1975) [Sov. Phys. Dokl. 20, 562 (1976)].

[19] S. Y. Lee, Spin Dynamics and Snakes in Synchrotrons (World Scientific, Singapore, 1997).

[20] V. Anferov, in Proceedings of the Conference on Polarized Protons at High Energies, edited by D. P. Barber and A. De Roeck (Report No. DESYProceedings-1999-03, 1999).

[21] A.W. Chao, in Proceedings of the Conference on Polarized Protons at High Energies (Ref. [20]).

[22] V. Ptitsin and Yu. M. Shatunov, in Proceedings of the Symposium on High Energy Spin Physics (SPIN96), Amsterdam, 1996, edited by C.W. de Jager, T. J. Ketel, P. J. Mulders, J. E. J. Oberski, and M. Oskam-Tamoezer (World Scientific, Singapore, 1996), pp. 516-518.

[23] E. D. Courant, BNL Report No. BNL-61920, 1995.

[24] G. H. Hoffstaetter, DESY Report No. DESY-96-05, 1996.

[25] G. H. Hoffstaetter, in Proceedings of the Symposium on High Energy Spin Physics (SPIN96), Amsterdam, 1996 (Ref. [22]).

[26] SPIN Collaboration and DESY Polarization Team, University of Michigan Report No. UM-HE-96-20, 1996.

[27] B.W. Montague, Phys. Rep. 113, 1 (1984).

[28] G.H. Hoffstaetter, in Increasing the AGS Polarization, edited by A. D. Krisch, A. M. T. Lin, and T. Roser, AIP Conf. Proc. No. 667 (AIP, New York, 2003), pp. 93-102.

[29] G.H. Hoffstaetter, University of Michigan Report No. UM-HE 99-05, 1999.

[30] H. Rose, Nucl. Instrum. Methods Phys. Res., Sect. A 258, 374 (1987).

[31] R. Degenhardt, Doctoral thesis, Darmstadt University of Technology, 1992.

[32] W. Wan, Ph.D. thesis, Michigan State University, 1995.

[33] G. H. Hoffstaetter, Nucl. Instrum. Methods Phys. Res., Sect. A 427, 275 (1998).

[34] G. H. Hoffstaetter, Nucl. Phys. A666-A667, 203c (2000).

[35] D. P. Barber, M. Vogt, and G.H. Hoffstaetter, in Proceedings of the European Particle Accelerator 
Conference (EPAC98), Stockholm, 1998 (IOP, London, 1998).

[36] G. H. Hoffstaetter, Ultramicroscopy 81, 111 (2000).

[37] Ch. Weißbäcker and G. H. Hoffstaetter, in Proceedings of the Conference on Polarized Protons at High Energies (Ref. [20]).

[38] K. Yokoya, DESY Report No. DESY-99-006, 1999.

[39] G. H. Hoffstaetter and M. Vogt, Sprint User's Guide and Reference Manual (DESY, Hamburg, 2002).

[40] D. P. Barber, K. Heinemann, G. H. Hoffstaetter, and M. Vogt, in Proceedings of the Symposium on High Energy Spin Physics (SPIN96), Amsterdam, 1996 (Ref. [22]).

[41] D. P. Barber, G. H. Hoffstaetter, and M. Vogt, in SPIN 2000: 14th International Spin Physics Symposium, edited by $\mathrm{K}$. Hatanaka, T. Nakano, K. Imai, and $\mathrm{H}$. Ejiri, AIP Conf. Proc. No. 570 (AIP, New York, 2001), pp. 751-755.

[42] D. P. Barber, G. H. Hoffstaetter, and M. Vogt, in Proceedings of the Conference on Polarized Protons at High Energies (Ref. [20]).

[43] K. Balewski et al., Nucl. Instrum. Methods Phys. Res., Sect. A 441, 274 (2000).

[44] M. Gentner, D. Husmann, C. Steier, R. Brinkmann, and Ya Derbenev, Nucl. Instrum. Methods Phys. Res., Sect. A 424, 277 (1999).

[45] R. Brinkmann, in Proceedings of the Conference on Polarized Protons at High Energies (Ref. [20]).

[46] D. P. Barber, G. H. Hoffstaetter, and M. Vogt, in the University of Michigan Report No. UM-HE 99-05, 1999. 